\title{
INSIGHT: SEMANTIC PROVENANCE AND ANALYSIS PLATFORM FOR MULTI-CENTER NEUROLOGY HEALTHCARE RESEARCH
}

by

\author{
PRIYA RAMESH
}

Submitted in partial fulfillment of the requirements for the degree of MASTER OF SCIENCE

Department of Electrical Engineering and Computer Science

CASE WESTERN RESERVE UNIVERSITY

January, 2016 


\section{CASE WESTERN RESERVE UNIVERSITY}

SCHOOL OF GRADUATE STUDIES

We hereby approve the thesis of

PRIYA RAMESH

candidate for the

Master of Science

degree*.

(signed)

Dr. Satya S. Sahoo, Ph.D.

(Chair of the committee)

Dr. Kenneth A. Loparo, Ph.D.

Dr. Martha Sajatovic, MD.

(date) $\quad$ November $5^{\text {th }}, 2015$

*We also certify that written approval has been obtained for any proprietary material contained therein. 
Copyright (C) Priya Ramesh January, 2016 All rights reserved. 


\section{TABLE OF CONTENTS}

TABLE OF CONTENTS $\quad$ iv

LIST OF FIGURES

LIST OF TABLES V vii

ACKNOWLEDGEMENT viii

$\begin{array}{ll}\text { CHAPTER 1. INTRODUCTION } & 10\end{array}$

CHAPTER 2. BACKGROUND 13

2.1 Managing Epilepsy Well Network 13

2.1.1 Introduction $\quad 13$

2.1.2 MEW database workgroup $\quad 16$

2.1.3 MEW Network survey results

2.1.4 A common terminology for epilepsy self-management 19

2.1.5 Standardization of data elements 20

2.1.6 Data curation workflow for MEW Network datasets 21

2.1.7 MEW Network database design and functionality 26

$\begin{array}{ll}2.2 \text { Research Studies } & 27\end{array}$

CHAPTER 3. METHODS 30

3.1. Semantic Integration Module using MEW Common Data Elements (CDEs) 33

3.2. Data Exploration and Query Module $\quad 34$

3.3. Ontology-based Inference Module $\quad 36$

CHAPTER 4. INSIGHT SOFTWARE DEVELOPMENT 38

4.1 Objective $\quad 38$

4.2 Agile Methodology 39

4.3 User Interface Development $\quad 41$

CHAPTER 5. INSIGHT SYSTEM 46

5.1 Ruby on Rails $\quad 46$

5.2 MVC Architecture $\quad 47$

5.3 System Environment 48

5.4 Development Environment 49

$\begin{array}{ll}\text { 5.4.1 MySQL } & 49\end{array}$

5.4.2 JavaScript 49

5.4.3 HighCharts API 50

5.4.4 Apache Web Server $\quad 50$

5.4.5 Ruby Version Manager (RVM) $\quad 50$

5.4.6 Phusion Passenger $\quad 50$

5.4.7 Cascading Style Sheets (CSS) 
5.4.8 Bootstrap

5.5 Insight Application Architecture $\quad 51$

5.6 Database Design 53

5.7 Notable features of Insight

5.7.1 Interactive Data Validation $\quad 53$

CHAPTER 6. USER INTERFACE OF INSIGHT

6.1 User Authentication $\quad 57$

6.2 Index Page $\quad 58$

$\begin{array}{lr}\text { CHAPTER 7. RESULTS } & 60\end{array}$

$\begin{array}{ll}\text { CHAPTER 8. DISCUSSION } & 62\end{array}$

$\begin{array}{ll}\text { CHAPTER 9. CONCLUSION } & 63\end{array}$

$\begin{array}{ll}\text { References } & 64\end{array}$ 


\section{LIST OF FIGURES}

Figure 2-1 Proposed architecture of the Managing Epilepsy Well (MEW) informatics

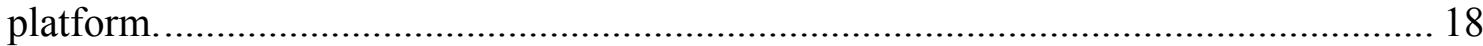

Figure 2-2 Screenshot of the Epilepsy and Seizure Ontology class hierarchy. ................... 26

Figure 3-1 Overview of the architecture and information flow in the Insight platform ........ 31

Figure 3-2 The two phases of cohort identification in the Insight platform. In Phase 1 (a), researchers use the study inclusion and exclusion criteria to select appropriate research studies. In Phase 2 (b), researchers use the MEW CDEs represented as visual query widgets to compose a cohort query and the results are displayed on the right of the screen listing all participant specific details in the research cohort............ 36

Figure 4-1 Agile Development for Insight............................................................ 40

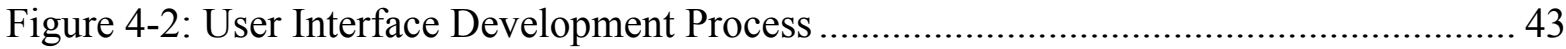

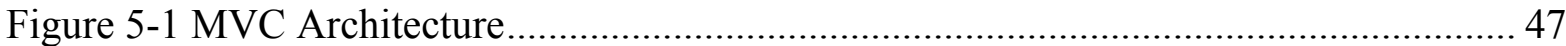

Figure 5-3 Data Presence Validation Example................................................................ 54

Figure 6-2 Sign Up Page: every new user is required to signup in order to gain access ....... 58

Figure 7-1 (a) The Insight data exploration module enables users to view the distribution of various data elements across the research studies (using pie chart and histogram). (b) The performance results for the ontology inference module using four example queries 


\section{LIST OF TABLES}

Table 1: Basic (Tier 1) elements in an integrated epilepsy self-management database. ........ 22

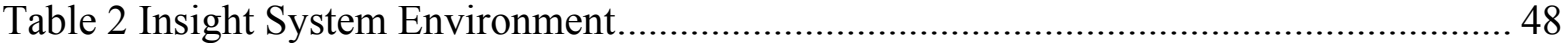




\section{ACKNOWLEDGEMENT}

I would like to thank all the people whose involvement was essential to the completion of my thesis.

First and foremost, this thesis would not have been possible without the constant support of my advisor, Dr. Satya Sahoo. His patient guidance has elevated the quality of my work and enriched my knowledge in the many aspects of this project. I was privileged to be a part of his research team.

The other member of my committee, Dr. Kenneth A. Loparo and Dr. Martha Sajatovic, have taken the time to review my work closely and I thank them for the invaluable feedback which has further enhanced my work.

I am grateful that this project was generously funded by National Institutes of Health (NIH), Grant No.1 U01EB020955-01. This gave me the opportunity to contribute significantly to the growing field of medical informatics.

Finally, I would like to thank my family and friends for their constant love and support. 
ix

\title{
Insight: Semantic Provenance and Analysis Platform for Multi-center Neurology \\ Healthcare Research
}

\author{
Abstract \\ by \\ PRIYA RAMESH
}

The Insight integrated platform is designed to support cohort identification, data exploration, and research queries from an integrated database with epilepsy study data from multiple centers using a self-service approach. The innovative features of Insight include: (1) the use of provenance metadata to select research studies with comparable inclusion/exclusion criteria, (2) visualization of data distribution at population level, and (3) interactive selection of query elements to provide essential contextual understanding for non-technical users in building complex cohort queries. Insight was developed to support semantic integration of heterogeneous data from multiple research studies in the Managing Epilepsy Well (MEW) Network. The MEW Network is funded by the U.S Center for Disease Control and Prevention (CDC) and consists of 8 centers across the U.S. Our long-term implementation and dissemination plans are moving towards deployment of Insight as a single point integrated portal for past and ongoing $\begin{array}{lll}\text { community-based } & \text { epilepsy self-management }\end{array}$ 


\section{CHAPTER 1. INTRODUCTION}

The rate of healthcare data generation and collection has rapidly increased in the last decade with wider adoption of electronic health records (EHR) systems, improved high-resolution imaging and capable recording instruments. Secondary analysis of this growing amount of healthcare data is allowing researchers to advance clinical research in many complex disease domains and is expected to lead to improvement in the quality of treatment as well as patient care (1). An essential component of secondary use of healthcare data is the need to support data integration and querying across different studies and multiple institutions. This will help ensure: (a) adequate statistical power with potentially broader representative samples for analysis, (b) maximal utilization of limited resources of funding agencies by leveraging investments in existing research studies, and (c) enabling researchers to plan future studies appropriately to address limitations of previous studies. For example, the Managing Epilepsy Network (MEW) Network, which consists of 8 centers across the U.S., conducts research studies in self-management techniques for epilepsy neurological disorder with funding from the U.S. Center for Disease Control and Prevention (CDC). Epilepsy is a chronic neurological disorder characterized by recurrent, unprovoked seizures affecting variety of mental and physical functions. Epilepsy affects about 50-60 million persons worldwide and is the most common serious neurological disease. In the United States, the CDC estimates that epilepsy affects about 2.2 million Americans with a greater prevalence than Parkinson's disease or multiple sclerosis (2).

The data collected by the different studies in the MEW Network are representative of a wide-variety of self-management techniques and different epilepsy 
patient populations, for example home-based intervention techniques for adults with epilepsy and co-morbid depression (3) and epilepsy patients with recent negative health outcomes such as hospitalization. Integrated analysis of these data can provide unique clinical research insights on various paradigms, such as treatment adherence practices (anti-epileptic medication), comparative effectiveness of epilepsy self-management techniques; or effect of socio-economic conditions on quality of life across the different sub-populations. The creation of an integrated database for the MEW Network, which can support cohort identification queries across different research studies, requires addressing both data heterogeneity and data access policies. However, this integration of data from multiple research studies, each with distinct study protocols and methods to assign values to data elements, makes it challenging to create cross-study research cohorts. For example, in the MEW Network each center uses different time period to assign a value to the "seizure frequency" data element (past 30 days, 2 months, or 1 year). The researchers need to be aware of this heterogeneity across studies during the query composition phase and use appropriate methods to reconcile this heterogeneity.

A large number of data integration tools are available in the healthcare informatics domain, for example the Informatics for Integrating Biology and the Bedside (I2B2) features a query interface consisting of clinical concepts that can be combined together to retrieve data from a data warehouse (4). Similarly, the Observational Medical Outcomes Partnership (OMOP) has developed informatics tools to integrate disparate data using the Common Data Model (CDM) (5). Both I2B2 and OMOP integration and analytical tools have been widely deployed to support clinical research data integration applications (6). Similarly, other data exploration and querying tools, such as Research 
Data explorer (RedX) have focused on EHR systems using a "query by example" approach (7). These tools are among the most popular data integration tools in clinical research informatics. However, these tools are focused on integration and querying of EHR data with limited usability for complex disease domains, such as epilepsy, which require use of specialized data elements at appropriate level of granularity. For example, existing query tools do not model various seizure categories and their associated brain regions, which make it extremely difficult for clinical researchers to create a cohort of patients with specific seizure category or anatomical location. In addition, we are not aware of any existing clinical informatics tool that incorporates "provenance metadata", which describes the inclusion and exclusion criteria of each participating study, to allow researchers to create patient cohorts from studies with comparable data values. Further, the MEW Network cohort identification tool requires use of standard vocabulary that models neurological disorder related terms at a fine level of granularity with appropriate coverage, which is not available in existing query tools.

In this thesis, I describe the development of Insight, a provenance-enabled study selection and cohort identification tool with rich data exploration and visualization features, which was developed as part of the MEW Network informatics initiative. I also describe the role of the Epilepsy and Seizure Ontology (EpSO) (8), which underpins Insight platform to support context-based cross-cohort queries with ontology-based reasoning. Our goal is to address the unmet challenges of provenance-enabled query development and cohort analysis over data from disparate studies and describe our experience in evolution of such tools as critical platforms for secondary analysis of biomedical data. 


\section{CHAPTER 2. BACKGROUND}

\subsection{Managing Epilepsy Well Network}

Epilepsy is a chronic neurological condition that requires active self-management to reduce personal and population burden. The Managing Epilepsy Well Network, funded by the US Centers for Disease Control and Prevention, conducts research on epilepsy self-management. There is an urgent need to develop an integrated informatics platform to maximize the secondary use of existing Managing Epilepsy Well Network data. The MEW Network has implemented multiple steps to develop an informatics platform, including: (a) a survey of existing outcome data, (b) identification of common data elements, and (c) an integrated database using an epilepsy domain ontology to reconcile data heterogeneity. The informatics platform enables assessment of epilepsy selfmanagement samples by site and in aggregate to support data interpretations for clinical care and ongoing epilepsy self-management research. The Managing Epilepsy Well informatics platform is expected to help advance epilepsy self-management, improve health outcomes, and has potential application in other thematic research networks.

\subsubsection{Introduction}

Epilepsy is a common serious neurological disorder that affects 65 million persons worldwide $(22,23)$. People with epilepsy experience repeated seizures that manifest as physical or behavioral changes. Epilepsy is treated with anti-epileptic medications to reduce or eliminate seizures; however, 30 percent of people with epilepsy continue to experience seizures in spite of medications $(24,25)$ and additional approaches are greatly needed. Epilepsy imposes substantial personal and societal burden. In the 
United States, annual cost of epilepsy is estimated to be US $\$ 12.5$ billion with about 85 percent of costs attributed to loss in productivity and in disability (26).

Epilepsy self-management is being increasingly explored as a potential approach to complement other existing treatments (27). Approaches that actively engage persons with epilepsy in taking care of their own health can potentially reduce the burden of epilepsy (28,33). In 2007, the Centers for Disease Control and Prevention (CDC) established the Managing Epilepsy Well (MEW) Network to develop, test, and disseminate epilepsy self-management interventions (34). Between 2007 and 2014, the MEW Network has incrementally grown to include six geographically distinct sites conducting epilepsy self-management research. This thematic research Network promotes collaboration on epilepsy self-management with a focus on gaps in knowledge or research related to public health practice.

Sites are tasked with developing and implementing a coordinated, applied research agenda; conducting research activities that promote epilepsy self-management and quality of life (QOL); and identifying and collaborating with public health, mental health, and other services agencies. The MEW Network has developed successful approaches for epilepsy self-management, including web-based epilepsy selfmanagement $(31,35)$ and community-based programs that address the common comorbidity, such as depression, in people with epilepsy $(29,36)$. These approaches have made innovative use of electronic, wireless, and mobile devices (e-Tools) to help people with epilepsy overcome the many barriers to care and self-management, such as lack of transportation and the stigma associated with having seizures in public $(34,37)$. In addition to use of remotely delivered interventions, the MEW Network taps into the 
growth of interest in patient-reported outcomes (38) similar to the Patient Reported Outcomes Measurement Information System (PROMIS)(39) program funded by the United States National Institutes of Health. Patient-reported outcomes that are highly relevant to people with epilepsy such as QOL and patient-perceived depression severity are a key component of many of the MEW Network initiatives and projects. The MEW Network centers have collected a large amount of research data since 2007 that represents important information about people with epilepsy and self-management. However, most self-management studies typically are not large, and there is a significant need to integrate datasets to answer questions that could otherwise not be answered with small individual study sample sizes. An integrated analysis of this large dataset will enable: (a) comparative effectiveness studies of self-management approaches, (b) cross-cohort queries to test various hypotheses, and (c) generation of insights to develop future selfmanagement programs. Many existing biomedical research networks are using informatics platforms for facilitating collaborative research and high performance distributed computing approaches for data managements $(40,42)$. In addition, secondary use of existing biomedical and health-care data has been identified as a priority by the Presidential Council of Advisors on Science and Technology (PCAST) report on health information technology (43).

However, data heterogeneity makes it difficult to integrate and query data across the Network. Reconciling data heterogeneity to develop data integration platforms has been an area of active research in computer science $(44,45)$. Data integration systems often use a common terminology system, which is mapped to terms from disparate data sources, to support both data processing and user queries (44). Ontology is an example of 
common terminology that uses formal knowledge representation language to model domain information for consistent and accurate interpretation by software applications $(46,47)$. The National Institutes of Health (NIH) funded National Center for Biomedical Ontologies (NCBO) lists more than 370 biomedical ontologies that have been developed to create common terminology systems in many biomedical and health-care domains. In this article, we describe the development of an ontology-driven MEW Network informatics platform with an integrated database to support future data analysis, cohort queries, and research studies that may be implemented across the MEW Network sites. This informatics infrastructure will significantly enhance the secondary use of MEW research data and ensure maximized returns on investments made by funding agencies to support epilepsy self-management research.

In this article, we describe the development of an ontology-driven MEW Network informatics platform with an integrated database to support future data analysis, cohort queries, and research studies that may be implemented across the MEW Network sites. This informatics infrastructure will significantly enhance the secondary use of MEW research data and ensure maximized returns on investments made by funding agencies to support epilepsy self-management research.

\subsubsection{MEW database workgroup}

Recognizing the need to develop an informatics platform that could analyze the data collected from disparate sources, the MEW Network convened a workgroup tasked with developing an integrated database strategy and testing the strategy in a proof-ofconcept exercise. The informatics platform development was initiated with seed funding from the Case Western Reserve University (CWRU) Prevention Research Center (PRC). 
The scope and functionality of the informatics platform was defined based on the results of a survey conducted in February 2013 with participation of all MEW Network sites. The survey collected data regarding the different categories of study data and the informatics approach used to manage the data in the Network. The survey used a questionnaire with seven query categories that required either a "yes/no" or a descriptive response. Completed surveys on 11 specific research studies were manually processed, and findings were assessed by the MEW workgroup.

\subsubsection{MEW Network survey results}

Most MEW research studies focused on comorbidities, treatment outcomes, and data required for predicting as well as evaluating the impact of self-management. Seizure frequency was collected in eight studies and emergency department data were collected in five. All studies collected data on seizure type, but only one collected data on seizure duration. Data on anti-epileptic drugs were collected in seven studies, and two collected data on psychotropic drugs. Other major categories of data included measures of QOL, cognition, depression, sleep quality, and general functioning. An important survey finding was the large number of disparate metrics used to collect research data across the MEW Network. For example, four different QOL metrics were used, with three studies using the Quality of Life in Epilepsy-31 (QOLIE-31) (48), two using the QOLIE-10 (49), one using the Neuro-QOL $(50,51)$, and one using internally developed Likert metrics. Similarly, 10 different cognitive measures and 5 depression measures were used. Heterogeneous measurement metrics represent a critical challenge to an integrated informatics infrastructure. 


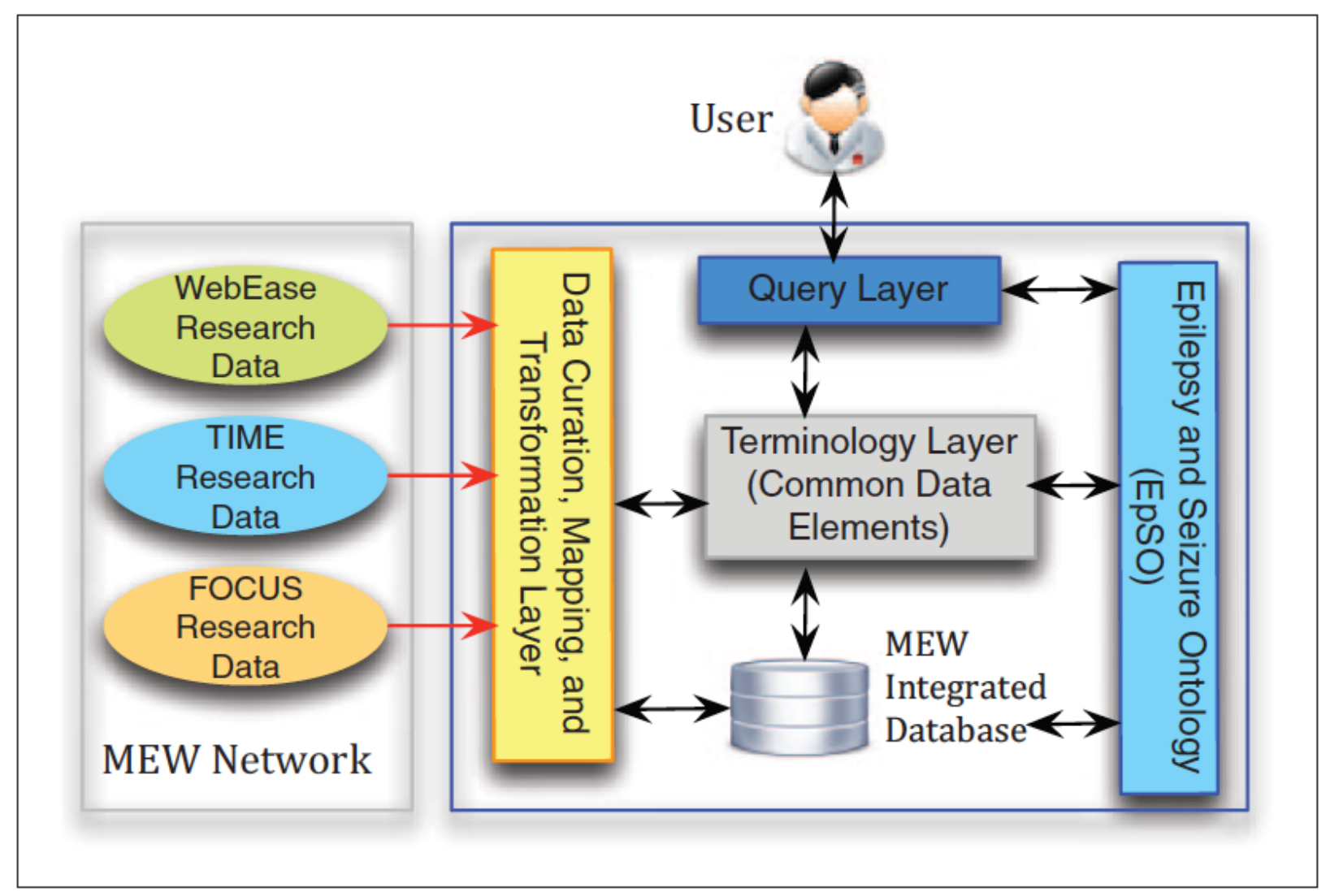

Figure 2-1 Proposed architecture of the Managing Epilepsy Well (MEW) informatics platform.

In addition to data heterogeneity, the survey found that only a few sites use a database management system (DBMS) to store and access data. Six of the studies used paper forms and nine used Microsoft Excel spreadsheets. Only one study used the Microsoft Access DBMS, and another study used REDCap (Research Electronic Data Capture) research software (52). Based on survey results and a presentation on the epilepsy informatics platform developed for clinical data management (53), the Network centers collectively agreed to participate in further work to integrate research data across participating sites. Figure 2-1 illustrates the architecture of the MEW Network informatics platform that consists of the following three components:

1. A common terminological system modeled as a domain ontology using a set of 
epilepsy self-management common data elements;

2. A data processing and curation pipeline that can reconcile heterogeneity in datasets from different sites using the epilepsy domain ontology as reference; and

3. An integrated database to support: (a) data analysis across different studies, (b) cohort building, and (c) cross-cohort queries.

The MEW informatics platform re-uses resources from an existing clinical informatics platform developed for epilepsy called the Multi-modality Epilepsy Data Capture and Integration System (MEDCIS) (54). The MEDCIS platform was developed to enable multi-center data integration and querying using the Epilepsy and Seizure Ontology (EpSO) as the common terminology system (55). We extended EpSO with epilepsy self-management terms to support the MEW informatics platform.

\subsubsection{A common terminology for epilepsy self-management}

A common terminology system is essential to develop an integrated database from disparate sources that will support user queries across different datasets. In the first step, we used the survey results, a literature review and group consensus to identify essential measurement constructs and metrics. The MEW informatics initiative included experts in epilepsy and mental health care, computer science, and public health. The workgroup members reviewed survey findings, the literature on data elements common to epilepsy self-management, and relevant large-scale epilepsy assessment and monitoring protocols. To efficiently use our limited resources to model the large number of epilepsy self-management terms, we used an incremental 3-tier phased development approach to develop the terminology system. In the Tier 1 phase, a core set of data elements (Table 1) 
were identified consisting of 16 variables that are frequently analyzed in epilepsy selfmanagement research studies. The Tier 1 variables describe basic demographic/clinical characteristics, health status, selected comorbidity measures, employment status, and seizure information. Existing epilepsy terminology references were used to define the variables, such as the Institute of Medicine's Report on "Epilepsy Across the Spectrum: Promoting Health and Understanding 2012,"(23) the recommended standards for epilepsy surveillance studies,35 the National Institute of Neurological Disorders and Stroke (NINDS) Common Data Elements (CDE) (57), and the Behavioral Risk Factor Surveillance System (BRFSS) Questionnaire (22).

Additional variables will be added in the Tier 2 phase, based on the specific requirements and Institutional Review Board (IRB) approvals of existing or new participating sites, while a comprehensive list of variables for epilepsy self-management will be constructed during the Tier 3 phase.

\subsubsection{Standardization of data elements}

A total of 42 data elements were used to describe the 16 Tier 1 variables, and these data elements were standardized and incorporated into the EpSO epilepsy domain ontology (55). EpSO uses the well-known four-dimensional epilepsy classification schema to model concepts of seizures, location of seizures, etiology, and related medical conditions $(58,59)$. In addition, EpSO models electroencephalography (EEG) and comprehensive drug information based on the US National Library of Medicine RxNorm standard (60). EpSO concepts are mapped to the NINDS CDE, which represents nine categories of terms describing imaging, neurological exam, neuropsychology, seizures,

and syndromes (57). EpSO has been used successfully to support standardized patient 
data entry in the Ontology-driven Patient Information Capture system (61), clinical text processing in the Epilepsy Data Extraction and Annotation system (62), and cloud-based electrophysiological signal processing in the Cloudwave platform (63). Hence, extending EpSO with epilepsy self-management terms will not only address data heterogeneity in the MEW informatics platform, but also support interoperability with other existing epilepsy informatics tools.

Figure 2-2 illustrates the modeling of the Tier 1 epilepsy self-management terms in EpSO, for example seizure count and the patient health questionnaire (PHQ). In addition to modeling the terms, EpSO annotates each class with additional information, for example PHQ item 7 (label) and Trouble concentrating on things, such as reading the newspaper or watching television (textual description). We plan to extend EpSO with additional terms during the Tier 2 and Tier 3 phases.

\subsubsection{Data curation workflow for MEW Network datasets}

In a proof-of-concept exercise, the MEW Network informatics platform was populated with research datasets from three studies: (a) the Web Epilepsy Awareness, Support, and Education (WebEase) project at Emory University (31), (b) the Targeted Illness Management for Epilepsy and Mental Illness (TIME) project at CWRU, and (c) the pilot of the FOCUS on Epilepsy program (FOCUS) at the University of Michigan (35). The WebEase study involved 148 individuals participating in an online epilepsy self-management study. The TIME study is an in-person, community-based intervention to improve mood and epilepsy outcomes in people with epilepsy and comorbid serious mental illnesses like schizophrenia, bipolar disorder and depression. The FOCUS study is testing a hybrid in-person and phone-based program that develops self-regulation skills in 
both adults with epilepsy and a key friend or family member who provides support.

Table 1: Basic (Tier 1) elements in an integrated epilepsy self-management database.

\begin{tabular}{|c|c|c|c|c|c|}
\hline & Variable & Scoring & & & Ref \\
\hline 1 & Gender & $\begin{array}{ll}\text { - } & \text { Male } \\
\text { - } & \text { Female }\end{array}$ & $\begin{array}{ll}\text { - } & \text { Unknown } \\
\text { - } & \text { Unspecified }\end{array}$ & - $\quad$ Other & $\mathrm{a}$ \\
\hline 2 & $\begin{array}{l}\text { Age in } \\
\text { years }\end{array}$ & & & & $\mathrm{a}$ \\
\hline 3 & Ethnicity & $\begin{array}{ll}\text { - } & \text { Hispanic or Latino } \\
\text { - } & \text { Not Hispanic or Latino }\end{array}$ & $\begin{array}{ll}\text { - } & \text { Unknown } \\
\text { - } & \text { Not reported }\end{array}$ & & $\mathrm{a}$ \\
\hline 4 & Race & $\begin{array}{l}\text { - } \\
\text { - } \\
\text { Native Hawaiian or other } \\
\circ \quad \text { Pacific Islander } \\
\text { - } \text { Black/African-American }\end{array}$ & $\begin{array}{ll}\text { - } & \text { Asian } \\
\text { - } & \text { White } \\
\text { - } & \text { Not reported } \\
\text { - } & \text { Unknown }\end{array}$ & & $\mathrm{a}$ \\
\hline 5 & Education & $\begin{array}{l}\text { What is the highest grade or year of school } \\
\text { you completed? } \\
\text { - } \quad \text { Never attended school or only } \\
\text { attended kindergarten } \\
\text { - Grades } 1 \text { through } 8 \text { (Elementary) } \\
\text { - Grades } 9 \text { through } 11 \text { (Some high } \\
\text { school) } \\
\text { grade } 12 \text { or GED (High school } \\
\text { College } 1 \text { year to } 3 \text { years (Some } \\
\text { college or technical school) } \\
\text { College } 4 \text { years or more (College } \\
\text { graduate) }\end{array}$ & & & $\mathrm{b}$ \\
\hline 6 & $\begin{array}{l}\text { Employmen } \\
\text { t } \\
\text { (Check all } \\
\text { that apply) }\end{array}$ & $\begin{array}{ll}\text { Are you currently ...? } \\
\begin{array}{l}\text { - } \\
\text { - }\end{array} \text { Amployed for wages } \\
\text { - } \quad \text { Out of work for } 1 \text { year or More } \\
\text { - } \quad \text { Unable to work }\end{array}$ & $\begin{array}{l}\text { - } \\
\text { - } \quad \text { A Student } \\
\text { - } \quad \text { Out of work for } \\
\text { less than } 1 \text { year } \\
\text { - } \quad \text { Retired }\end{array}$ & & $\mathrm{b}$ \\
\hline
\end{tabular}




\begin{tabular}{|c|c|c|c|c|c|}
\hline 7 & Income & $\begin{array}{l}\text { Is your annual household income from all } \\
\text { sources- } \\
\text { - } \quad \text { Less than } \$ 24,999 \\
\text { - } \$ 25,000-\$ 49,999 \\
\text { - } \$ 50,000 \text { or greater }\end{array}$ & & & $\mathrm{b}$ \\
\hline 8 & $\begin{array}{l}\text { Household } \\
\text { composition }\end{array}$ & $\begin{array}{l}\text { How many children less than } 18 \text { years of } \\
\text { age live in your household? } \\
\text { How many adults } 18 \text { and older live in your } \\
\text { household? }\end{array}$ & & & $\mathrm{b}$ \\
\hline 9 & $\begin{array}{l}\text { Relationshi } \\
\mathrm{p} \\
\text { status }\end{array}$ & $\begin{array}{l}\text { Are you ...? } \\
\begin{array}{ll} & \text { Married } \\
\text { - } & \text { Never married } \\
\text { - } & \text { A member of an unmarried } \\
& \text { couple }\end{array}\end{array}$ & $\begin{array}{l}\text { - } \quad \text { Divorced } \\
\text { - } \quad \text { Separated }\end{array}$ & $\begin{array}{l}\text { - } \quad \text { Widowed } \\
\text { - } \quad \text { Refused }\end{array}$ & $\mathrm{b}$ \\
\hline 1 & $\begin{array}{l}\text { Age of } \\
\text { onset } \\
\text { of seizures }\end{array}$ & $\begin{array}{l}\text { At what age were you diagnosed with } \\
\text { epilepsy? }\end{array}$ & & & $\begin{array}{l}\mathrm{N} / \\
\mathrm{A}\end{array}$ \\
\hline 1 & $\begin{array}{l}\text { Frequency } \\
\text { of seizures }\end{array}$ & $\begin{array}{l}\text { - Yes } \\
\text { - No } \\
\text { If yes, how many? Number of seizures in } \\
\text { last } 12 \text { months }\end{array}$ & & & \\
\hline 1 & $\begin{array}{l}\text { Types of } \\
\text { seizures } \\
\text { (Check all } \\
\text { that apply) }\end{array}$ & $\begin{array}{cl}\text { - } & \text { Generalized seizure } \\
\text { - } & \text { Generalized nonconvulsive } \\
\text { - } & \text { Don't know/not sure } \\
\text { Source of data: } \\
\text { - Self-report } \\
\text { - } \text { Medical record review }\end{array}$ & $\begin{array}{ll}\text { - } & \text { Focal seizure } \\
\text { - } & \text { Focal seizure } \\
\text { Insight/loss of } \\
\text { consciousness } \\
\text { - Unclassified } \\
\text { seizure }\end{array}$ & & $\begin{array}{l}\mathrm{N} / \\
\mathrm{A}\end{array}$ \\
\hline 1 & $\begin{array}{l}\text { Health } \\
\text { status }\end{array}$ & $\begin{array}{l}\text { Would you say that in general your health } \\
\text { is- } \\
\text { - Excellent } \\
\text { - Gery good } \\
\text { - Good }\end{array}$ & $\begin{array}{l}\text { - } \\
\text { - } \quad \text { Pair } \\
\text { - } \quad \text { Don't know/not }\end{array}$ & & $\mathrm{c}$ \\
\hline
\end{tabular}




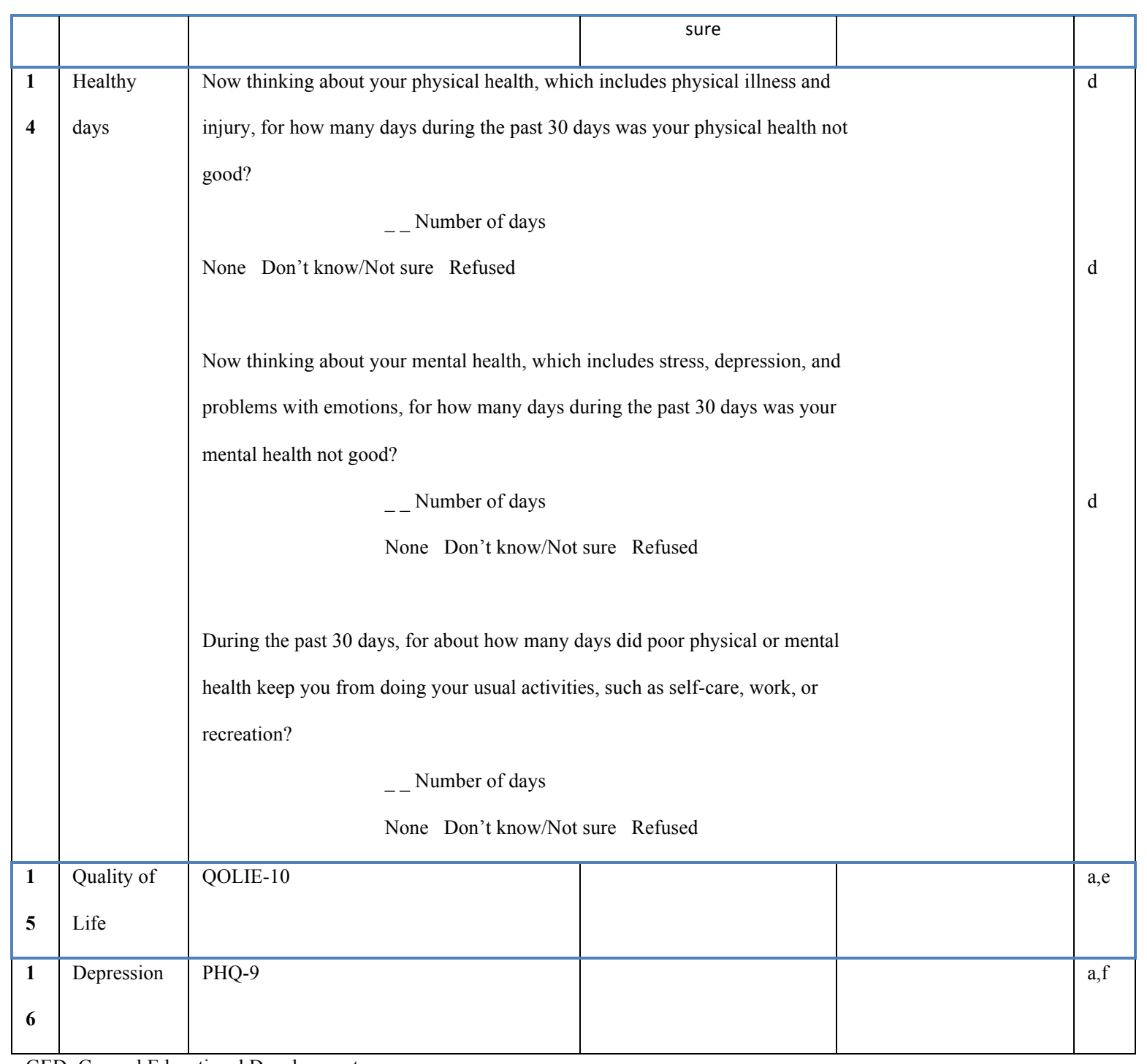

GED: General Educational Development.

${ }^{a}$ National Institute of Neurological Disorders and Stroke (NINDS) Common Data Elements (CDE).

${ }^{\mathrm{b}}$ Centers for Disease Control and Prevention (CDC).1

${ }^{\mathrm{c}}$ Behavioral Risk Factor Surveillance System (BRFSS): Section 1.

${ }^{\mathrm{d}}$ BRFSS: Section 2 .

${ }^{\mathrm{e}}$ Quality of Life in Epilepsy-10 (QOLIE-10).28

${ }^{\mathrm{f}}$ Patient Health Questionnaire-9 (PHQ-9): Kroenke K, Spitzer RL and Williams JB. The PHQ-9: validity of a brief depression severity measure. J Gen Intern Med 2001; 16: 606-613.

We developed three data flows corresponding to each research study as part of the data curation, mapping, and transformation layer to extract, transform, and load (ETL) 
data from each of the three research studies (Figure 2-1). The ETL workflows consisted of multiple data processing phases.

The first step generates mapping between study-specific variables and the MEW common terminology system, which involves reconciling differences in both data values and interval values used to categorize the data elements. For example, the first three interval values for the education variable in TIME correspond to "Never attended school," "grades 1 through 8," and "grades 9 through 11." The WebEase study used a single interval of "School from 1 through 11;" hence, education values of "1," "2," and " 3 " in TIME correspond to a value of " 3 " in WebEase, but the inverse in not true. To address this issue, mappings were defined to map value "1" in WebEase to value " 3 " in the integrated database.

Another example of data heterogeneity involves the use of value " 1 " in the FOCUS dataset in contrast to value "2" used in the MEW common data elements to represent "Hispanic or Latino" participants. There are no completely automated techniques to reconcile this "semantic heterogeneity" in data integration systems (64); hence, the appropriate mappings were manually generated for the MEW datasets. In the next step, these mappings are used to extract and automatically transform each research dataset, using common terms that are mapped to EpSO.

The data processing step assigns each study participant a unique identifier and annotates the corresponding data value with provenance information describing the source study. Provenance metadata, which describes the source of data (65), allows the MEW informatics platform to filter query results based on the research study. The ETL workflows were developed in a modular manner, which allows the MEW informatics 
platform to incrementally add new research datasets without disrupting user interactions over the deployed database. In future work, we propose to deploy the study-specific workflows as Web services (66), which are Web applications that can be remotely invoked by the MEW informatics platform as part of a Service Oriented Architecture to populate the integrated database.

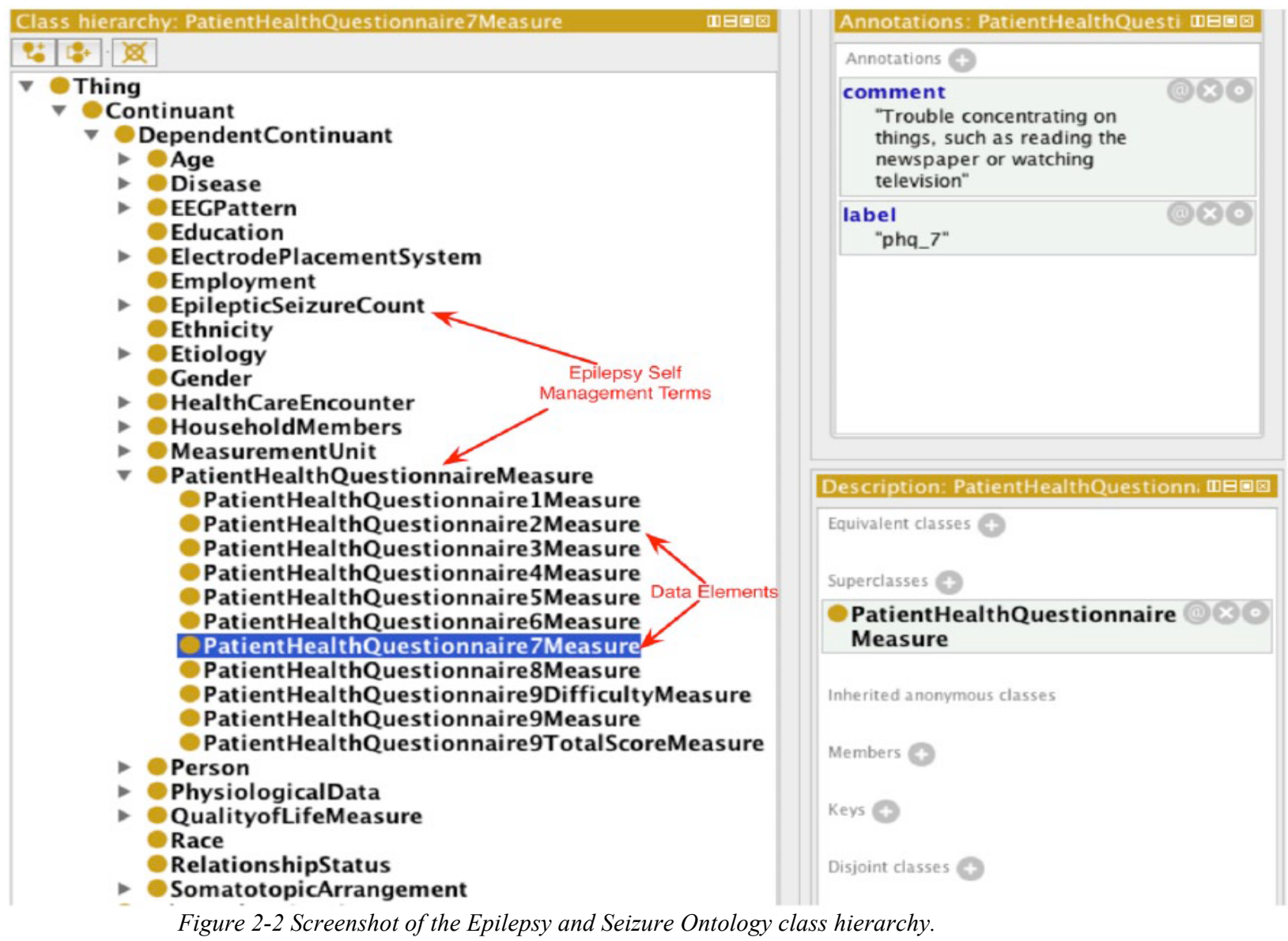

\subsubsection{MEW Network database design and functionality}

The integrated database used a relational data model to store the data. The integrated database is currently hosted on a local protected computer drive and will be 
moved to a dedicated and secure MEW computing environment in the future to make it available to the MEW Network. We are currently developing an intuitive user interface to query the database that will re-use many resources of the existing Visual Aggregator and Explorer user interface deployed for the MEDCIS platform. The user interface will feature query widgets corresponding to study variables that can be composed to express queries (67), a result display section with "live count" of patient cohorts, and visualization tools that can support statistical analysis. We used the open source MySQL DBMS for the integrated database, together with the Structured Query Language (SQL), to compose and execute the queries described in this article. As the volume of the data increases with integration of additional MEW research datasets, we will migrate the database to open source high performance column-based HBase DBMS (68). Two categories of queries were executed over the integrated database to retrieve: (a) values for seven variables describing demographic and clinical data that are related to QOL values for all participants across the three research studies and (b) values corresponding to all 42 common data elements divided into three sets for each study to describe the total sample represented by the integrated database. The query results were analyzed using standard statistical techniques.

\subsection{Research Studies}

The Insight platform integrated data from three completed research studies in the MEW Network. We briefly describe these three research studies as background for the data integration and analysis described in the subsequent sections. 


\section{The FOCUS research study:}

The aim of the Enhancing Self-regulation and Social Support in Adults with refractory Epilepsy study was to pilot test a self-management intervention for adults with refractory epilepsy and a key member of their social support network. The intervention combined helping people with epilepsy develop skills for daily self-management, training persons who are providing social support to those with epilepsy, and providing salient information to both the people with epilepsy and their support person. This was accomplished by conducting a daylong retreat, followed by a series of telephone coaching sessions to reinforce, practice and reflect upon the skills learned. Key outcome measures that were collected included epilepsy-related quality of life, positive affect and well-being, depressions, healthy days, and epilepsy self-management behaviors.

\section{The TIME research study:}

TIME (Targeted Self-Management for Epilepsy and Mental Illness) was a randomized, controlled trial, intended for adults with epilepsy and a serious mental illness (SMI), such as severe depression, bipolar disorder, and schizophrenia. It consisted of a selfmanagement program based upon a social cognitive model and featured psychoeduction, behavioral modeling and group support. The program included 12 weekly in-person group sessions, led by a nurse educator and peer educators, that covered topics such as information on epilepsy-SMI interactions, personalized goal setting, medication adherence and dealing with the "double stigma" of having both epilepsy and SMI. Participants were evaluated at screen/baseline, and at 12 and 16 weeks, and outcome measures included change in SMI symptoms, epilepsy-related quality of life, treatment adherence, functional status, sleep pattern, social support and self-efficacy. 


\section{The WebEase research study:}

The WebEase (Epilepsy Awareness, Support and Education) study was designed to test a web-based self-management program. The program consisted of three interactive modules: medication, stress and sleep-management; in addition, the website contained a discussion board, fact sheets, quizzes and daily polls. The study objectives were to determine if older adolescents and adults who participated in the WebEase program had improved epilepsy-related knowledge, medication adherence, stress management, sleep time and quality, and quality of life. Participants completed a baseline assessment and were randomly assigned to the intervention or wait list control condition. Participants assigned to the intervention condition began WebEase immediately after completing the baseline assessment, while those in the wait-list control condition waited 6 weeks and completed a second survey before beginning the WebEase program. Both groups completed a third survey 12 weeks after the baseline assessment. 


\section{CHAPTER 3. METHODS}

The MEW Network aims to advance epilepsy self-management research and to reduce comorbidity, such as depression and anxiety in persons with epilepsy. The MEW Network has actively and successfully engagement with local community members, advocacy groups such as the Epilepsy Foundation and professional organizations such as the American Epilepsy Society (9). The MEW Network centers conduct research studies related to epilepsy management from the perspective of individuals who have epilepsy. The data collected within each study represents valuable information about a variety of parameters about epilepsy and self-management techniques and an integrated database with support for cohort identification will be an important resource for the wider epilepsy research community. The Insight platform is designed to address this need by using a "self-service" approach that allows users to explore the data distribution in different studies through intuitive visualization features and interactively create research cohorts that take into account the provenance information about each data source. 


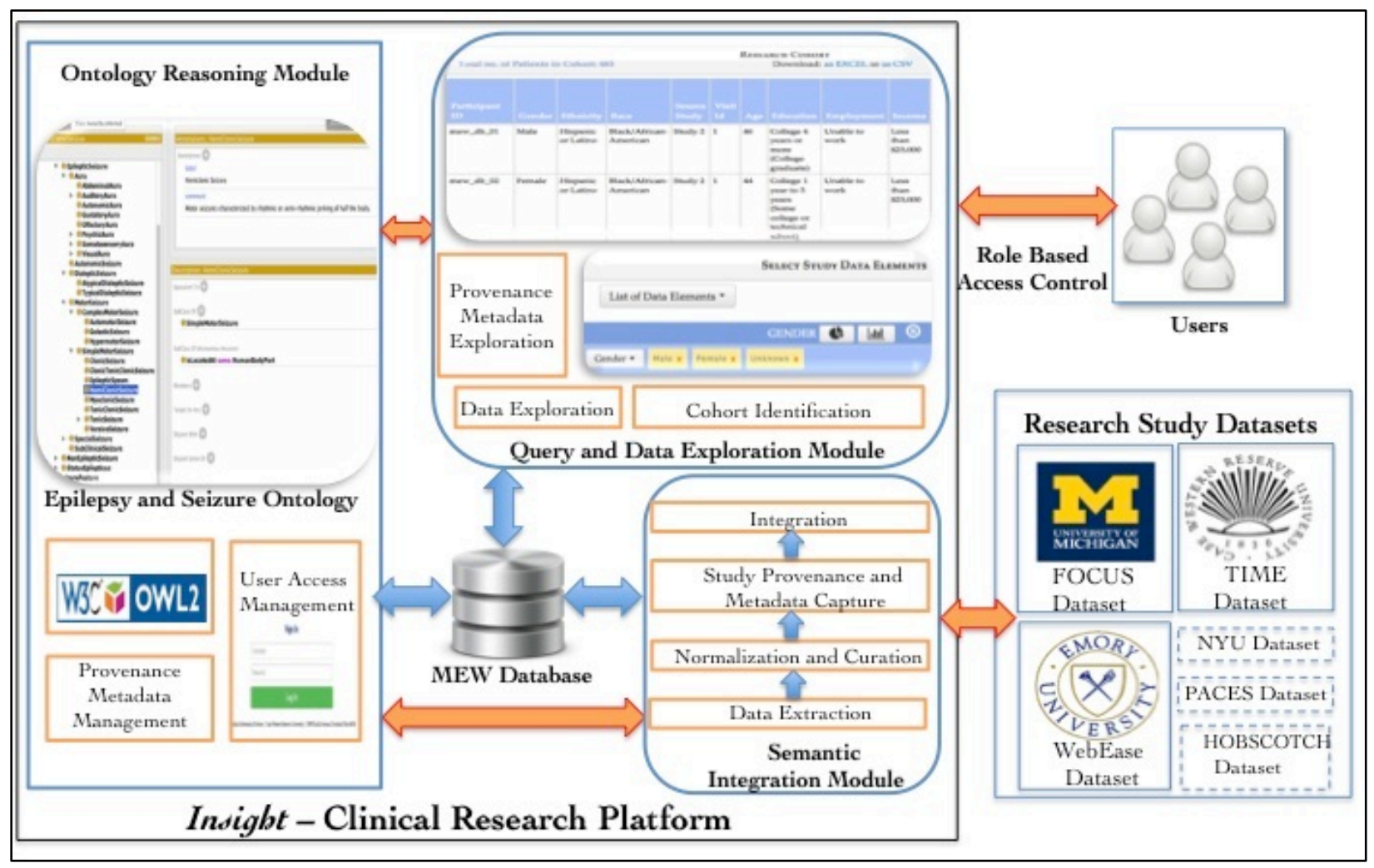

Figure 3-1 Overview of the architecture and information flow in the Insight platform

The design of the Insight platform incorporated information about experience and requirements of the MEW Network members in data management, which was collected through a survey conducted in 2013 (10). We also took into account the lessons learned from the query mediation process used to retrieve research data from clinical data warehouses (7). The key design features of Insight are: (1) a two-phase query approach that consists of provenance-enabled research study selection followed by the use of common data elements for cohort identification, (2) interactive query composition with real-time visualization of data distribution of individual data elements at population level for different studies, and (3) use of ontology reasoning during query execution for 
semantic matching of participant records with query constraints. Figure 3-1 illustrates the three components of the Insight platform:

1. Semantic Integration module: The semantic integration module uses an extract transform load (ETL) pipeline to integrate study data from different MEW centers using a set of MEW Common Data Elements (CDE), which are modeled in EpSO as ontology concepts. The transformed and integrated data is annotated with ontology concepts from EpSO.

2. Data Exploration and Query module: This module implements a two-phase querying strategy by allowing researchers to select comparable research studies based on their inclusion and exclusion criteria followed by use of "query widgets" with visualization features for distribution of data values.

3. Ontology-based Inference module: Using OWL2 reasoning over EpSO, this module infers and retrieves all the participants that satisfy the cohort query constraints by reconciling syntactic and semantic heterogeneity.

Insight uses EpSO as an epilepsy domain ontology to support data integration and query execution. EpSO is being developed as part of a National Institutes of Neurological Disorders and Stroke (NINDS)-funded Center for Sudden and Unexpected Death in Epilepsy (SUDEP) Research (CSR), which aims to use clinical data from 14 epilepsy centers in the U.S and the U.K to identify SUDEP biomarkers. EpSO uses the wellknown four-dimensional classification of "epileptic seizures and epilepsies" to model seizure types, etiology, anatomical region associated with seizure occurrence, and related medical conditions (ㅁ). EpSO uses the World Wide Web Consortium (W3C) recommended Web Ontology Language (OWL2) to represent the terms, their properties, 
and define class-level restrictions to allow automated reasoning. EpSO re-uses brain anatomy concepts from the Foundational Model of Anatomy (FMA) (11), the Neural ElectroMagnetic Ontologies (NEMO) (12), and drug information from the RxNorm (13). EpSO models the data elements used in the MEW Network to support semantic integration of data from different studies.

\subsection{Semantic Integration Module using MEW Common Data Elements (CDEs)}

A survey of existing data collection methods used in the MEW Network identified a large number of disparate measurement metrics used to describe different categories of self-management data. For example, four different Quality of Life (QOL) metrics were used in the MEW studies, including QOLIE-31 (14), QOLIE-10 (15), and Neuro-QOL (16). Similarly, ten cognitive measures and five depression measures were used to collect participant information in the MEW Network. This data heterogeneity presents a significant challenge to data integration and cohort identification. Therefore the MEW database workgroup defined a set of common data elements (MEW CDEs) to harmonize heterogeneous study data that re-used many existing epilepsy specific terminological systems. These included: (1) the Institute of Medicine's report called "Epilepsy across the

Spectrum: Promoting Health and Understanding 2012" (17), (2) the recommended standards for epilepsy surveillance studies (18), (3) the NINDS Common Data Elements (NINDS CDE) (19), and (4) the CDC Behavioral Risk Factor Surveillance System (BRFSS) questionnaire (20). The MEW CDEs are being created using a phased approach. In Phase I, the CDEs were selected to represent demography, quality of life metrics, 
seizure types, frequency of seizures etc. and in Phase II the CDEs will cover seizure severity, stress, medication adherence, and sleep quality, among other measure.

The MEW CDEs are modeled as formal ontology classes in EpSO and these ontology classes are used by the ETL pipelines to process and semantically annotate the study data. The ETL pipelines uses data mapping rules to reconcile the seizure frequency values to a common time period. As part of the ETL process, the individual study names and participant identification were replaced by a standardized identification values, such as Study 1 for research studies. This step was requested by the MEW database workgroup to reduce the possibility of mapping participants to specific research studies. The ETL pipelines implement multi-stage complex data mapping and transformation steps to reconcile heterogeneity in data values, interval values, and labels. For example, research studies collected "seizure frequency" data over different time periods (past 30 days or 6 weeks or 4 months), which made it difficult to standardize the seizure frequency values extracted from different studies. If data values cannot be transformed into standard format, the differences are explicitly noted as metadata information that the users can access through the visual interface during data exploration and cohort identification. The data exploration and query interface also allows users to select research studies based on their inclusion and exclusion criteria, which is modeled as provenance metadata in the integration database during the semantic integration phase.

\subsection{Data Exploration and Query Module}

Research studies use different inclusion and exclusion criteria to select study participants and it is necessary for researchers to take into account the context of each study when creating research cohorts from different studies. The inclusion and exclusion 
criteria represent critical provenance metadata about the research studies that allow researchers to more accurately interpret analysis results from cross-study cohorts. Therefore, the Insight Data Exploration and Query module allows researchers to follow a two-phase provenance-enabled cohort identification approach. In the first phase, users select the appropriate inclusion and exclusion criteria to identify comparable research studies. Figure 3-2(a) illustrates the "Research Study Selection" section of the Insight user interface that allows researchers to select two MEW Network studies, Study 1 and Study 2, based on specified inclusion and exclusion criteria. Further, researchers can also view the complete study protocol by selecting the "View Study Protocol" button and download the complete list of inclusion and exclusion criteria. In the second phase, researchers can identify a cohort by interactively composing a cohort selection query using CDE widgets with values that can be selected from multi-level drop down menu. Figure 3-2(b) illustrates the CDE widgets, the user selected values, and drop-down menu for seizure types that are mapped to the ontology classes in EpSO.

In addition, users can also view the distribution of different data values during the cohort query composition, which allows users to visualize the overall distribution of values for that $\mathrm{CDE}$ at a population level and select appropriate cohort query constraints. Researchers specifically requested the development of this data exploration feature during the development of Insight, which provides two standard distribution views using the pie chart and histogram layout. These views allow researchers to help understand the most common values for a study variable, for example Figure 7-1 shows that "African American" is the most common value for "Race" in the WebEase and FOCUS studies, which can be used to decide the cutoff values for variables in the cohort identification. In 
addition, the views allow researchers to compare data elements values across different study populations, which can be used to select additional data elements to improve the sensitivity or specificity of the cohort identification query. Once a user submits the cohort identification query, the Insight ontology-based inference module uses reasoning over the class hierarchy to retrieve all relevant data values from the integrated database.

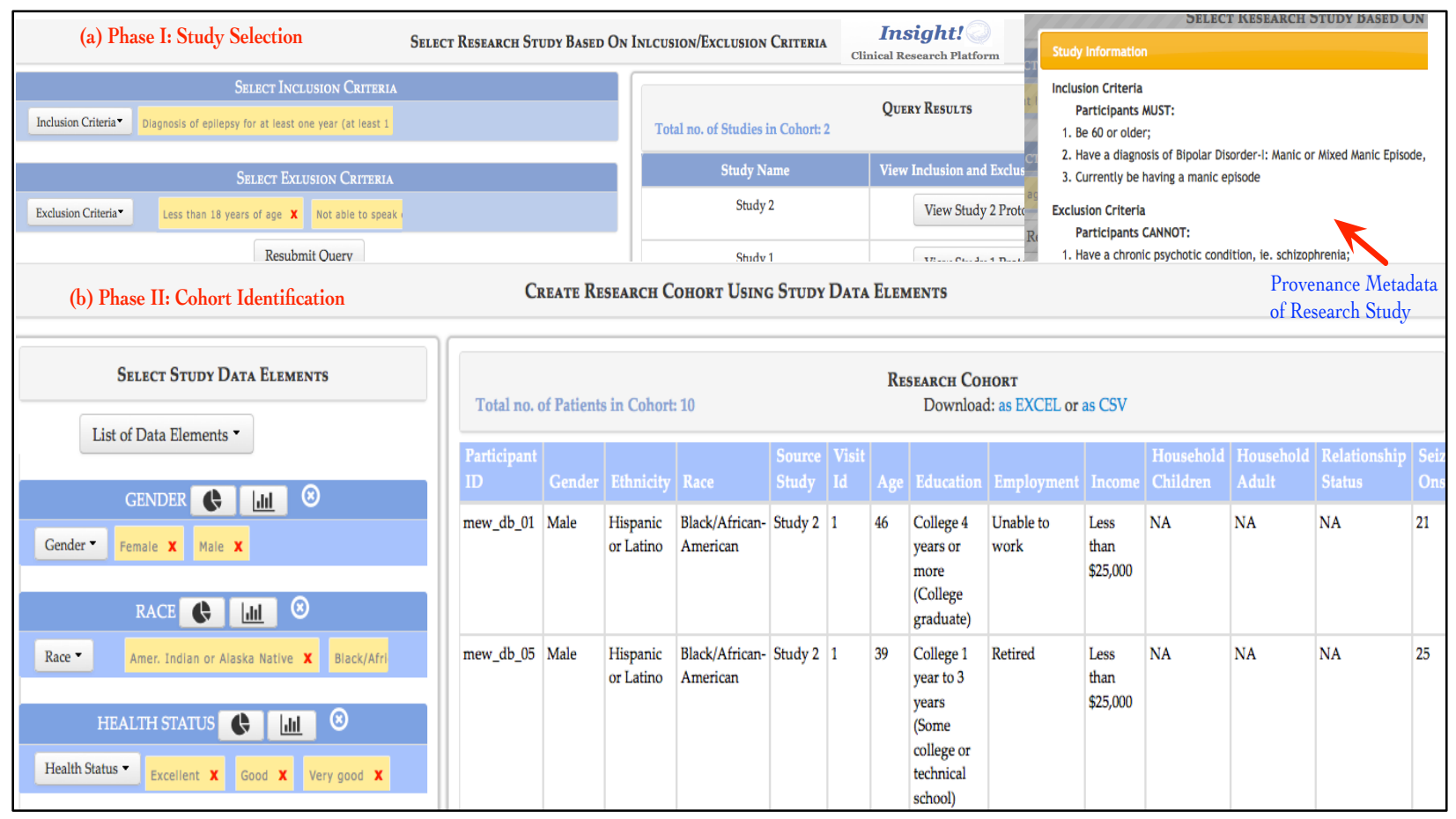

Figure 3-2 The two phases of cohort identification in the Insight platform. In Phase 1 (a), researchers use the study inclusion and exclusion criteria to select appropriate research studies. In Phase 2 (b), researchers use the MEW CDEs represented as visual query widgets to compose a cohort query and the results are displayed on the right of the screen listing all participant specific details in the research cohort.

\subsection{Ontology-based Inference Module}

The semantic annotation of data in the integrated database allows the use of ontology reasoning to "expand" the cohort query expression using subsumption reasoning 
to identify all subtypes of an ontology class. For example, a cohort identification query may use the seizure type "aura" (a particular type of seizure), however if the study uses more specific types of seizure, such as "somatosensory aura" or "visual hallucination", then the query execution algorithm needs to infer that both "somatosensory aura" and "visual hallucination" are subtypes of "aura". The use of ontology reasoning in Insight ensures that the resulting research cohort includes data for all appropriate participants. Similarly ontology reasoning allows Insight to reconcile heterogeneity in drug brand names, drug ingredients, and categorization of drugs by their indications, for example anti-epileptic drugs (AED), neuroleptic drugs, and anti-depressants. As we discussed earlier, EpSO models drug information by re-using the terms from RxNorm (13), which are linked to epilepsy disease classes using OWL2 class-level restrictions.

The Insight platform uses the open source OWLAPI (21) to parse the EpSO OWL file and support ontology reasoning during query execution. In addition, the ontology module extracts additional information about each ontology class using $r d f s: l a b e l$ and rdfs:comment properties, which users can access during cohort identification for reference. The ontology module features a semi-automated mechanism to update the ontology version using a Web service to access and retrieve the latest version of EpSO from a remote server. The use of ontology reasoning requires additional computations during cohort identification and as part of our evaluation we tested the performance of Insight using four cohort identification queries. 


\section{CHAPTER 4. INSIGHT SOFTWARE DEVELOPMENT}

\subsection{Objective}

The successful and efficient management of software applications has been a constant topic of research. Software applications have been divided into three groups based on their completion and performance, the three groups are successful, challenged and impaired. The main reasons for a software application to fail are as follows:

1. Incomplete requirement fulfillment: Projects that are flawed and cancelled as the specified features and functions are incomplete.

2. Inadequate user involvement: The involvement and participation of the user in the application has a major effect on the final result and fate of the application.

3. Neglected agile process adoption: Projects that fail to adopt the agile methodology of software development end up with an application that is not fully functional.

To avoid the above-mentioned challenges in software development, this thesis describes the software development used for developing Insight, which focuses on involving the customer during the development phase at every step to make sure that the requirements are being met. The use of agile development methodology ensured that Insight used cost-effective and time-effective development process.

Agile User Interface Development is used in this thesis to accomplish the task at hand. This ensures a smooth and track able development process. The Agile User Interface Development unlike other software development methods emphasizes on user interaction and allows researchers and doctors to test and assess the system before implementing any database management system. This kind of constant and incremental 
assessment of the system obtained from clinical experts and researchers who have exceptional understanding about Insight, provide valuable information to the software developer who can use it to improve the system until the required perfect product that meet all the requirements is obtained. This constant customer interaction ensures that the developers as well as customers are on the same page, which ensures that the final product is error free and fully functional. This kind of development is both time and cost effective. The incremental user interaction accelerates the development speed and reduces communication complexities between people from different backgrounds. The agile methodology is highly beneficial when there is a chance that the requirements constantly change. The Waterfall methodology uses a sequential approach to development and testing, which reduces the effectiveness of the application when it has to accommodate changes. Agile methodology, on the other hand ensures cost and time effectiveness.

\subsection{Agile Methodology}

The software development of Insight assimilated the fundamental concepts of the agile methodology. Figure 4-1 describes the main principles that define the agile manifesto. The main values are: (1) Interaction between business people and developers, (2) Frequent delivery of software, (3) Constant and continuous attention to technicality

and design, (4) Maintaining a constant development speed (5) Regular adaptation to changing requirements. The first step in the development of a successful application involves the understanding of the objectives and requirements by working closely with the experts. This provides sufficient information to the development team to make reasonable and achievable strategies and plans for each iteration. The development team 
then proceeds by creating a prototype and sensible design, which is used during the development phase. The functionality after being implemented is tested by the development team and then presented to the clients, who provide valuable feedback of the interface. The client's feedback and comment's are recorded. They are then used constructively to improve the system. The system is continuously improvised until the clients are pleased with the application and usability of the interfaces. The steps involve in agile methodology are shown in Figure 4-1.

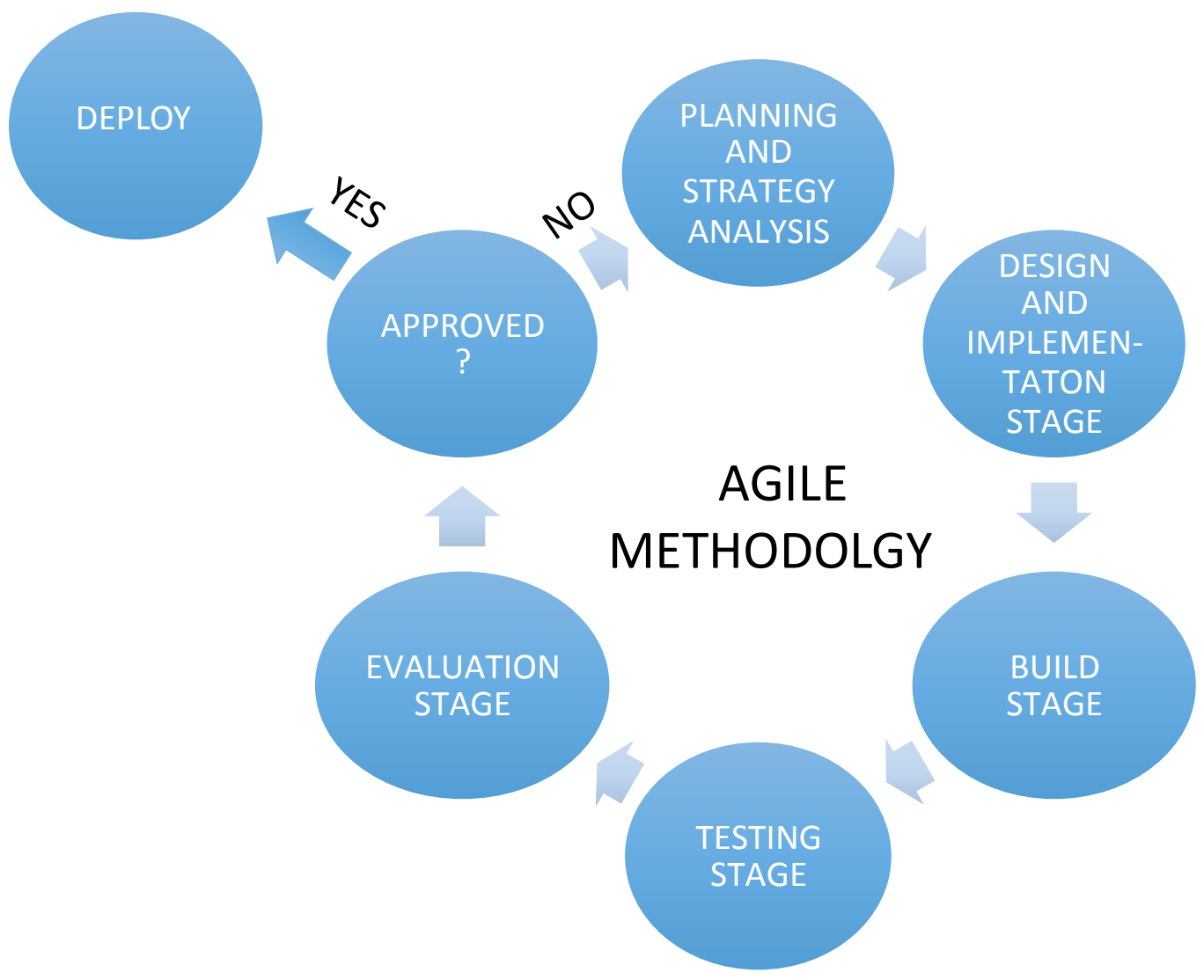

Figure 4-1 Agile Development for Insight

The software development of Insight incorporated the principles of the agile methodology to ensure the collaboration between the client and developers. During the development, meetings with clients were given high priority to ensure that the 
requirements were being rightly implemented, confusion with respect to functionalities were reduced and functionalities that were not feasible to implement were discussed before the developers actually started coding. This technique proved to be beneficial to both the development team as well as client, as it ensured that the clients were always aware of what was being implemented and the stage of the project. The client's involvement in the development phase ensured that they contributed in the design and construction of the application. This form of weekly interaction between the development team and client proved to be very advantageous, it allowed changes in the requirements to be implemented with ease and helped in the development of a successful end product, which was intact with all functionalities. The main advantage of agile methodology is the fact that it ensures the creation of an open-ended and accommodating system. All changes in the requirement or scope of the project can be easily implemented at any stage in the development cycle. The use of agile strategy allows Insight to be extremely flexible and accommodative of unforeseen requirements. The software development process ensured that the requirements and changes were documented for future reference and this allowed Insight to be an application that can easily accommodate sudden and unpredictable changes which would not have been possible without the use of agile strategies and methods.

\subsection{User Interface Development}

An interactive user interface is required for the effective management and storage

of clinical data. A user interface provides a way of intuitively analyzing patient data and provides a medium through which a user can interact with the data. A user interface that is designed well and is rich with required functionalities is very useful in to the client. 
Agile methodology used in the User Interface Development ensures that the user is given an interface that is rich in design and is easy to use. The development of such an interface is divided into three main phases as shown in Figure 4-2.

The agile methodology in the user interface development uses a balanced approach to create an interface that is not only functional but also usable. It ensures that interface exposes the task and functionalities exactly in a way which is specified by the user.

This approach first develops a system that imitates all the required features and functionalities without interacting with the data model. The system behaves in a manner that is very similar to the final product without actually interacting with the data stored in the database management system. The meetings ensure that the prospective users of this system test and assess the system providing useful constructive feedback. The Role based access feature allows users with different roles to perform different operations on the system. This method allows the development team to understand the level of satisfaction of the users who interacted with the system and make the necessary changes if required. This is followed up with numerous revisions and refactoring of the system based on the user feedback to create an application that is in accordance with the specifications keeping the final objective in mind.

The modules that make up the system are implemented separately in compliance to the agile methodology. The development of each module is accompanied with testing in parallel. All the developed modules are combined together to form the final system. The system is tested again as one unit to ensure that all the functionalities are not only 
working individually but are also working when combined together with other functionalities. The agile user interface development comprises of the following phases:

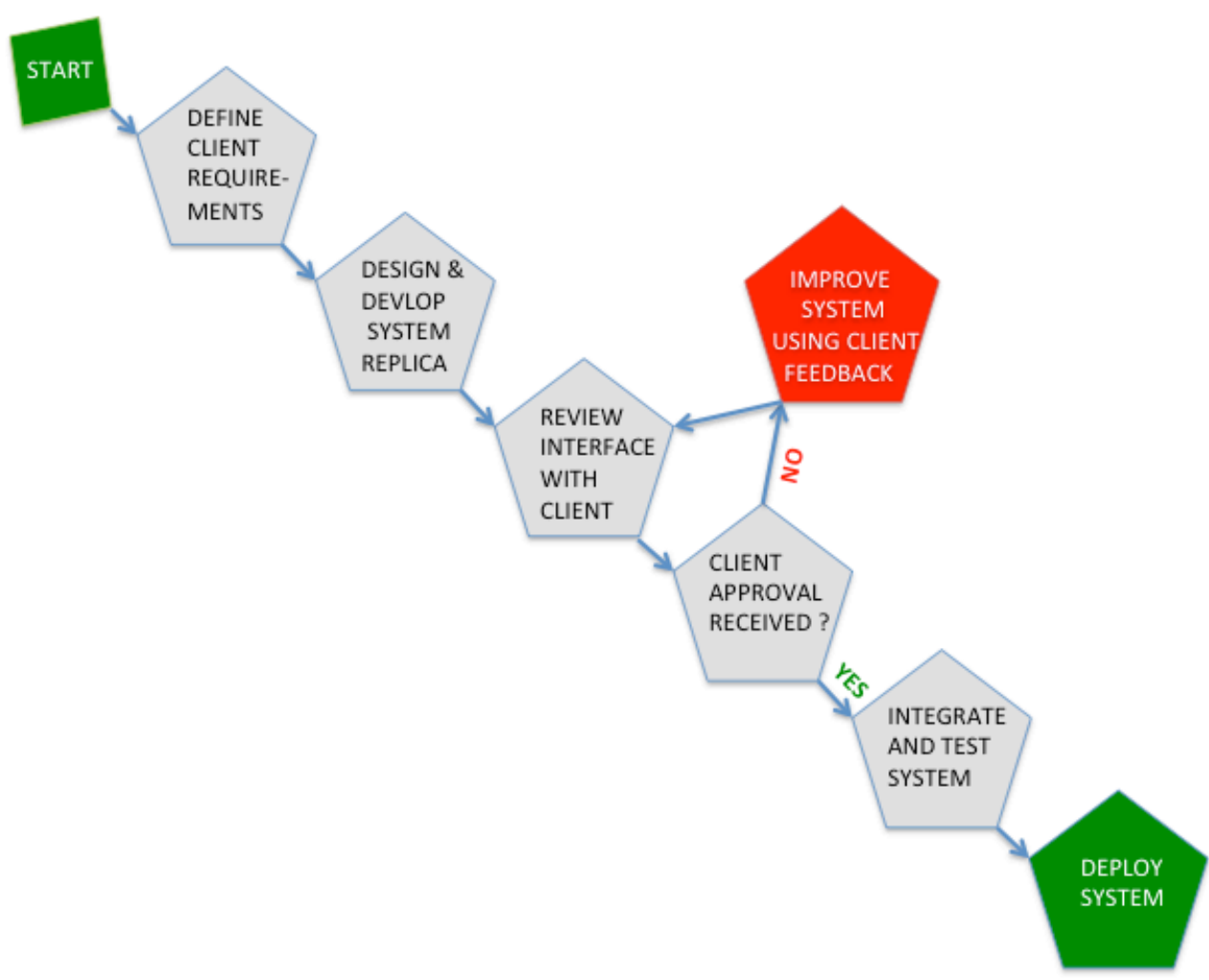

Figure 4-2: User Interface Development Process

The three main phases involved in the development of a user interface are as follows: (1) Design, (2) Implementation, and (3) Testing.

The Design phase consists of the following steps that ensure that the interface being developed is fully functional. The steps involved are:

a) Identify functional requirements: This involves establishing the high-level requirements and goals of the application. 
b) User analysis: This involves understanding the need and expectations of the user.

c) Conceptual Design of the system: This involves understanding the operations that application must support.

d) Technicality involved in the design of the system: Identify the logical and technical background required to implement the system.

e) Physical Design of the system: Identify the platform on which the application will be developed and deployed.

The implementation phase makes use of the information collected during the design phase to develop the required application. The steps involved in this phase are as follows:

a) Design the application's prototype: This involves the creation of a model that imbibes all the requirements and functionalities mentioned by the client that fully imitates the final product to be created.

b) Development of the application: The user interface is constructed keeping in mind the functionalities and requirements. This is implemented in a manner that maximizes the user experience and minimizes cost and time overhead.

The Testing phase is the most important phase in the development of a user interface. It ensures that the application is created in accordance with the user specifications. It involves the following steps: 
a) Usability Testing: Different users who understand the needs of the system test the application in different situations. This ensures that the application doesn't fail in any situation.

Application and Accessibility Testing: The user interface is testing on different platform, using different automated testing tools. 


\section{CHAPTER 5. INSIGHT SYSTEM}

\subsection{Ruby on Rails}

Insight is developed using Ruby on Rails Model-View Controller (MVC) architecture. "Rails' is a web-application framework that includes everything needed to create database-backed web applications according to the Model-View Controller (MVC) pattern" (69).

"Rails' is a web application development framework written in the Ruby language. It is designed to make programming web applications easier by making assumptions about what every developer needs to get started. It allows you to write less code while accomplishing more than many other languages and frameworks. Experienced Rails developers also report that it makes web application development more fun" (70).

“The Rails philosophy includes two major guiding principles:

- Don't Repeat Yourself: DRY is a principle of software development which states, "Every piece of knowledge must have a single, unambiguous, authoritative representation within a system." By not writing the same information over and over again, our code is more maintainable, more extensible, and less buggy (70).

- Convention Over Configuration: Rails has opinions about the best way to do many things in a web application, and defaults to this set of conventions, rather than require that you specify every minutia through endless configuration files “(70). 
Insight code is developed using ruby on rails' increases the flexibility, reduces maintenance difficulty, but also enables Insight to be used consistently with the ever changing clinical environment. The use of ruby on rails' during implementation makes it a platform that can be used universally to analyze patient data across various sites and platforms.

\subsection{MVC Architecture}

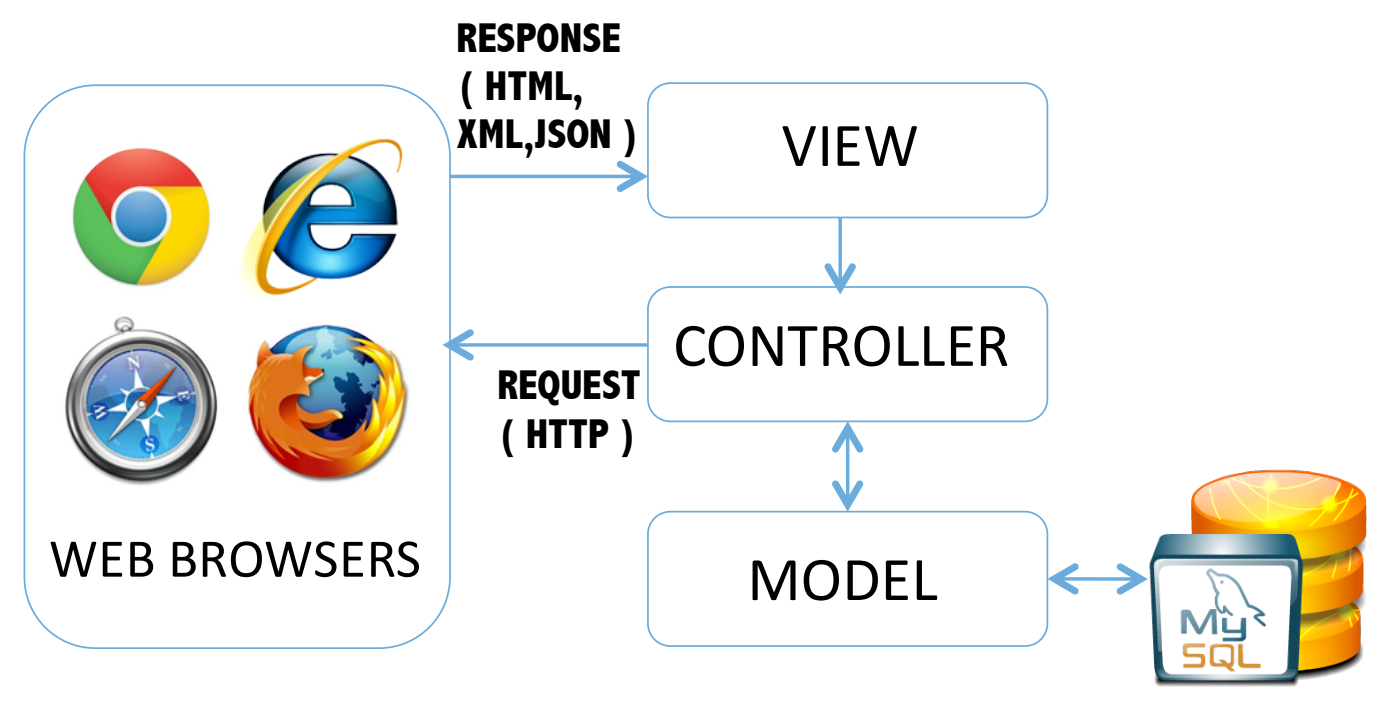

Figure 5-1 MVC Architecture

The MVC architecture is a popular software design pattern used for implementing and developing web based software applications. The model view controller architectural pattern is made up of three main parts as shown in Figure 5-1. The three main parts are:

- Model - The model represents any kind of data. It is responsible for the storage of data. 
- View - The view can be described as the visualization unit. It is responsible for displaying all or some of the data stored in the models and making it available to the user.

- Controller - The controller is responsible for controlling the interaction and data flow between the model and the view. The controller receives the input from the user, performs required operations on it and then used it to either retrieve data from the model or change the data stored in the model.

The MVC architectural pattern used in Insight, helped in maintaining and managing the complex code and logic involved in creating Insight.

\subsection{System Environment}

Table 2 illustrates the system environment required by Insight. All the requirements listed below have been thoroughly tested.

Insight is compatible with web browsers like Google Chrome, Safari and Firefox on machines with Mac OS, Ubuntu or Microsoft Windows as the operation systems

Table 2 Insight System Environment

\begin{tabular}{|c|c|}
\hline Name & Requirement \\
\hline Ruby & 2.0 .0 \\
\hline Rails & 4.0 .3 \\
\hline
\end{tabular}




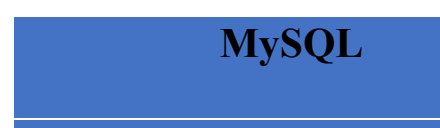

Web Browser
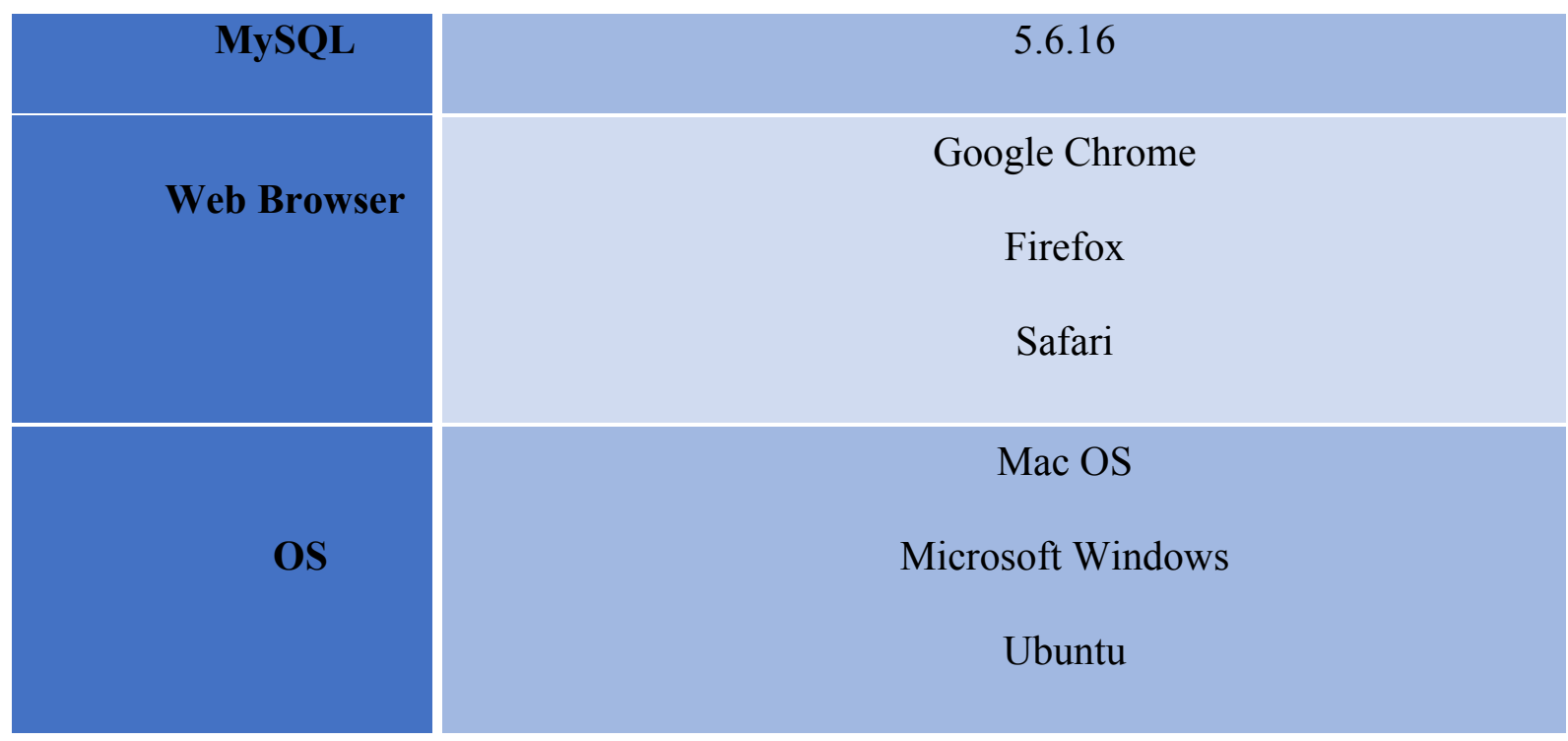

\subsection{Development Environment}

\subsubsection{MySQL}

"My Structured Query Language (MySQL) is the world's most popular opensource database. MySQL can help you deliver high performance, scalable database applications" (71). Insight uses the version 5.6.x.

\subsubsection{JavaScript}

It is a programming language which is used to keep the webpages interactive and intuitive. Including JavaScript is used mainly to perform dynamic tasks and increase the performance of the applications. Insight uses JavaScript to maintain the static pages and create a complex responsive web application. 


\subsubsection{HighCharts API}

"Highcharts is a charting library written in pure JavaScript, offering an easy way of adding interactive charts to your web site or web application. Highcharts currently supports line, spline, area, areaspline, column, bar, pie, scatter, angular gauges, arearange, areasplinerange, columnrange, bubble, box plot, error bars, funnel, waterfall and polar chart types“"(72). Insight uses the bar and pie charts to analyse the common data elements.

\subsubsection{Apache Web Server}

Apache web server usually runs on Unix based operating systems (such as Linux, Solaris, Digital UNIX, and AIX). It was used to host our Insight system. This is fast, reliable, lightweight, secure and stable.

\subsubsection{Ruby Version Manager (RVM)}

"RVM is a command-line tool which allows you to easily install, manage, and work with multiple ruby environments from interpreters to sets of gems" (73).

\subsubsection{Phusion Passenger}

"Phusion Passenger is a multi-language (Ruby, Python, Node) web \& app server which can integrate into Apache and Nginx. It is a web server and an application server" (74). We use version 4.0.45. 


\subsubsection{Cascading Style Sheets (CSS)}

"Cascading Style Sheets (CSS) is a simple mechanism for adding style (e.g., fonts, colors, and spacing) to Web documents. Nearly all browsers nowadays support CSS and many other applications do, too "(75).

\subsubsection{Bootstrap}

"Bootstrap is the most popular HTML, CSS, and JS framework for developing responsive, mobile first projects on the web. Bootstrap makes front-end web development faster and easier. It's made for folks of all skill levels, devices of all shapes, and projects of all sizes " (76).

\subsection{Insight Application Architecture}

Insight is a typical example of a three layered architecture used commonly in web applications. Figure 5-2 illustrates this commonly used web application architecture. The figure demonstrates the three-layered architecture that is composed of presentation, business and data layers. The presentation layers represents the user interface and logic components associated with the user interface. The business layer includes the workflow and business components. It contains the logic associated with authentication and information handling. The data layer handles data management. The data layer provides connection to external database systems. The presentation layer runs on the clients system, while the business and data layer are present in the remote host server. 
Insight can be run on different web browsers like Safari, Google Chrome and Firefox. The web server used in Insight is Apache; it is used to manage HTTP requests sent from web browsers. MySQL is used as database management system, which stores and manages the patient data required for the application. The client accesses Insight using a web browser, this creates a connection between the presentation layer and the business layer. Insight makes sure that only an authenticated user can login to the application. This confirms that only authenticated users can manipulate patient data stored in the database. The role based access functionality ensures that different users can access patient data based on their role.

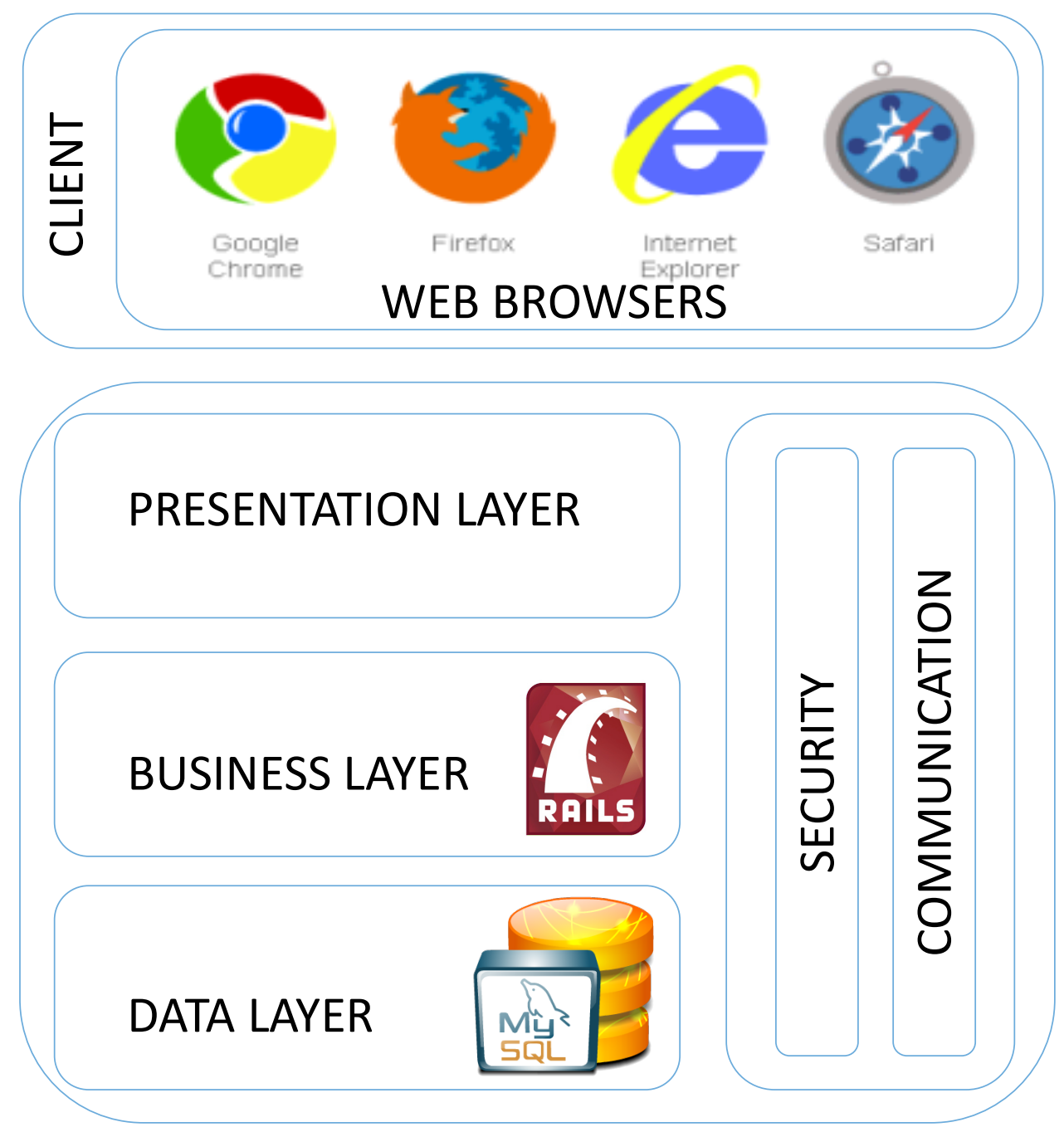




\subsection{Database Design}

Participant data is divided into 3 cores in Insight. They are: administrative core, clinical core and configuration cores. There are a total of 10 data models for the 3 cores. Administrative core contains participant demographic and registration information. Clinical core includes multiple participant visit information. Configuration core contains all the models used to populate the interface.

\subsection{Notable features of Insight}

\subsubsection{Interactive Data Validation}

Insight provides interactive data validation for user input to avoid, identify and correct errors and inconsistencies in participant information. A variety of implicit and explicit validation rules are used to check for correctness and meaningfulness of user input. The validation rules used in Insight include:

1. Data presence check: This rule validates that the required fields are not left blank. For example, in the "Sign up" form, used to create new user, fields like 'First name', 'Email' and 'Password' cannot be empty. Otherwise, a warning message is displayed below the invalid field as shown in Figure 5-3. 


\section{Insight!}

Clinical Research Platform

\section{Sign Up}

\section{Form is invalid}

- Password can't be blank

- First name can't be blank

- Email can't be blank

First name

Email

Password

Password confirmation

Create New User

Figure 5-3 Data Presence Validation Example

2. Data range and limit constraint: In many data fields, valid input values have to be within a pre-determined range. For example, the data element 'age' has a range of minimum age and maximum age. A predefined range is set for these fields. Therefore, the fields that violate the predefined validation rule are marked red as shown in Figure 5-4. 


\section{Select Study Data Elements}

List of Data Elements -

\section{AGE (?}

Min Age 1 :-Max Age $30 \mathrm{pr}:$

Figure 5-4 Placeholder and Range Validation Example

3. Data type conformance: Each data entry field is usually associated with a simple data type (integer, float, Boolean, date, time), which can be used to automatically apply data type conformance checks. In the example below the Household Composition field has been defined as an integer type which allow the electronic system to automatically check data entry for conformance against the type definition as shown in Figure 5-5.

4. Data uniqueness guarantee: Specific fields inclusion/exclusion cannot be repeated in Insight. If a user inputs a value that already exists, a warning message would be displayed

\section{Select Study Data Elements}

\section{List of Data Elements -}

\section{HOUSEHOLD COMPOSTIION (ㄱ)}

\section{Number of Children less than 18 years}

abc :

Number of Adults 18 and older 
at the top of the page.

5. Restricted value sets. Data entry fields may be associated with a set of permissible values. Drop down lists and radio buttons are used to restrict the selection. In Figure 5-6 the "Inclusion Criteria" variable is restricted to one of 8 values; the user can enter no other data value.

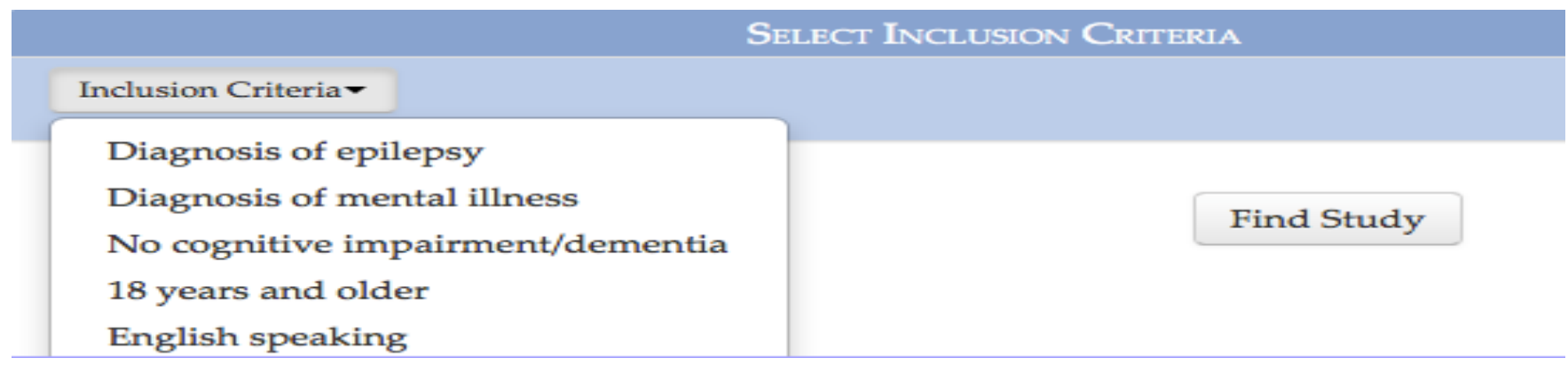

Figure 5-6 Restricted Value Set Example

The implementation of the data validation rules, (1) decreases the chance of potential human typing errors, (2) reduces the expensive and time-consuming post-entry artificial examination and correction; (3) increases data capturing accuracy which enables faster decision making. 


\section{CHAPTER 6. USER INTERFACE OF INSIGHT}

\subsection{User Authentication}

Insight contains participant personal information and medical record, which under no circumstances should be revealed and maliciously accessed by unqualified personnel. To ensure the security of the data, the authentication procedure requires all the users to successfully log in to the system before getting access to the data as shown in Figure 6-1. A new user needs to provide personnel information like an email address, log in ID and contact info to successfully submit a registration for the use of Insight (Figure 6-2).

\section{Insight!}

Clinical Research Platform

Sign In

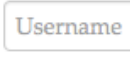

Password

$\log \operatorname{In}$

Medical Informatics Division | Case Western Reserve University | 10900 Euclid Avenue Cleveland Ohio 44106 
Warning message will be displayed if the user makes any error or inappropriate format.

User's registration needs to be proved by any of the system administrators. Only authorized users can have access to Insight.

\section{Sign Up}

\section{First name}

\section{Email}

\section{Password}

\section{Password confirmation}

\section{Create New User}

Figure 6-2 Sign Up Page: every new user is required to signup in order to gain access

\subsection{Index Page}

Upon log in, the user is presented with the default root page that consists of the query interface process, which consists of two phases as shown in the figure below. The first phase allows the user to obtain studies based on the selected inclusion criteria's. Once the studies are obtained, the user can modify his criteria selection and resubmit the query. 
Then second query phase allows the user to create the research cohort based on the selected data elements and their values. Selecting different data elements and resubmitting the query can modify the research cohort.

\section{Insight}

Clinical Research Platform

Logged in as MEW Admin. Log out $\mathrm{C}$

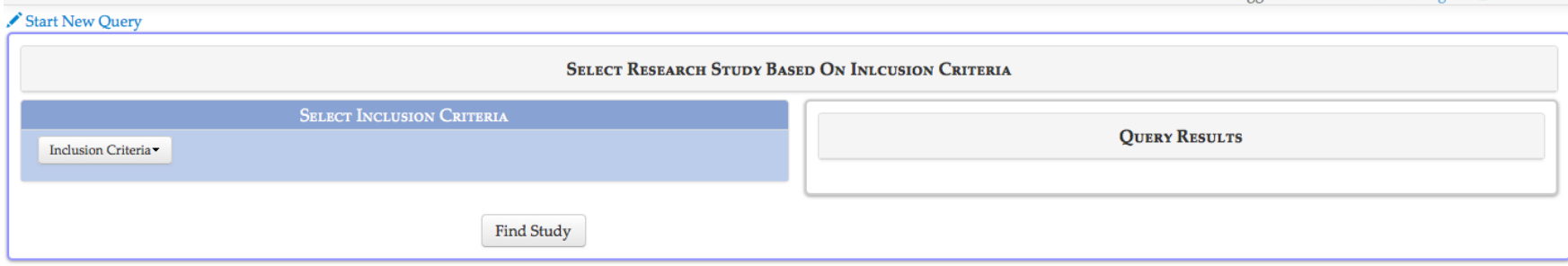

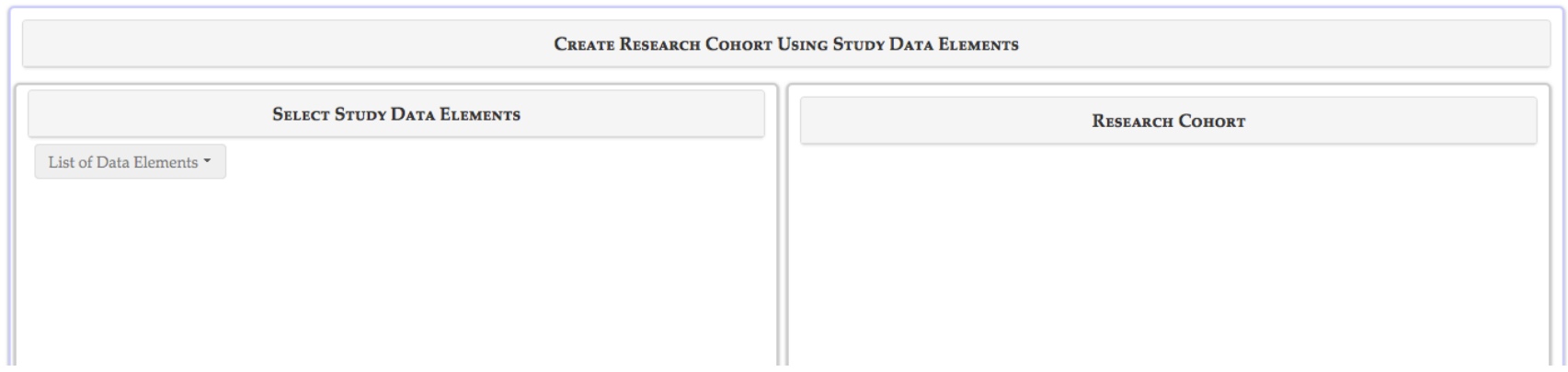

Figure 6-3 Index Page: The Query Interface 


\section{CHAPTER 7. RESULTS}

The Insight platform was developed to meet the design objectives of a provenance enabled interactive cohort identification system and it successfully completed the three major functions identified in the design. Users can directly navigate to data elements of a particular participant in a research cohort, which consists of data from multiple MEW Network research studies. Researchers can either download the research cohort data as an MS Excel spreadsheet or comma separated values (CSV) for subsequent analysis (shown in Figure 7-1(b)). Users can also download the complete data for a single study during the study-selection phase. The user interface supports dynamic modification of both provenance metadata constraints and cohort query constraints, which allows users to adjust the research cohort according to their study requirements. Currently, we are conducting an extensive multi-site user evaluation study to collect feedback on the features and functions of Insight.

In addition, we performed an evaluation of the computational cost associated with use of ontology reasoning over EpSO for cohort identification using four example queries. The four queries involved reasoning over the class hierarchy of "Aura" (Query 1), both “Aura" and "Motor Seizure” (Query 2), "Simple Motor Seizures” (Query 3), and "Complex Motor Seizure" (Query 4). The evaluation involved processing of the datasets

on a standalone server machine with Intel $\mathrm{Xeon}(\mathrm{R}) 2.20 \mathrm{GHz}$ processor, 8GB main memory, a 1536KB L2 cache, and 15MB L3 cache. Figure 7-1(b) shows that use of ontology reasoning during cohort identification leads to only a small increase in query performance time with little impact on response time of Insight. These experiment results 
support the design decision to use ontology reasoning for improving the accuracy of data retrieval during cohort identification without any decrease in response time of Insight.

Insight was developed using an agile software engineering process with frequent feedback from clinical users with multiple development iterations. The user interface was developed using Ruby-on-Rails, which supported quick implementation of different features and ease of deployment. The use of web services to support automated update of the ontology inference module with the latest version of EpSO OWL file conforms to many aspects of service oriented architecture. This feature also supports additional extension of Insight to interface with other domain ontologies in the future.

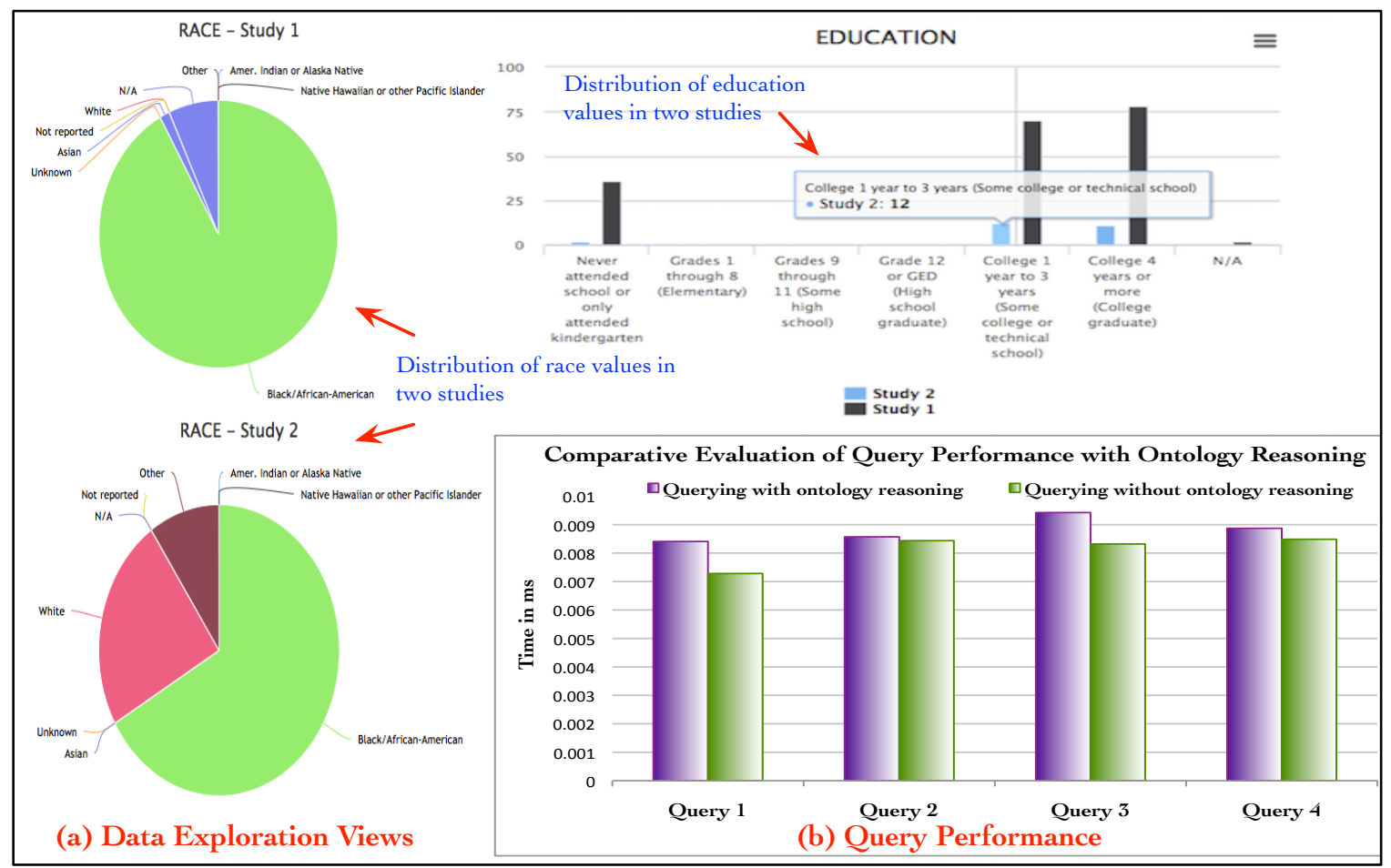

Figure 7-1 (a) The Insight data exploration module enables users to view the distribution of various data elements across the research studies (using pie chart and histogram). (b) The performance results for the ontology inference module using four example queries 


\section{CHAPTER 8. DISCUSSION}

The Insight platform focused on providing a self-service environment for researchers to conduct cohort identification and data analysis queries across multiple research studies. This was a differentiating objective of Insight and it provides the MEW Network access to integrated data on epilepsy self-management collected from the various sites. Initial usability evaluations show that the two-phase provenance-enabled study selection followed by cohort identification provided researchers with a better understanding about the distribution of data values across different studies. The data exploration views were considered to be important by the users and the use of ontology reasoning to ensure quality of resulting cohorts was also seen an a significant function by users.

We are currently adapting the database component to new developments, such as the OMOP CDM, which will ensure greater interoperability of Insight with existing clinical informatics tools. We are specifically interested in developing an I2B2 plug-in that will allow the large user community of I2B2 to access epilepsy-specific cohort identification tool. This will ensure greater availability and dissemination of disease domain focused cohort identification to the wider clinical research informatics community. Further, we are also identifying additional data analytics features, such as various statistical measures, which can be made available to users. The computation and visualization of mean, variance, and related statistical measures will allow researchers to quickly gain an understanding about the characteristics of the data values within and across research studies. This will enable them to identify research cohorts in a more informed manner. 


\section{CHAPTER 9. CONCLUSION}

The development of an integrated cohort identification platform for the multicenter MEW Network project has the potential to enable more effective secondary use of existing clinical research data to advance epilepsy self management techniques. The Insight platform uses ontology-based data integration, data exploration, and highly interactive cohort identification features to support researchers in creating appropriate research cohorts across multiple research studies. 


\section{REFERENCES}

1. Safran C, Bloomrosen, M., Hammond, Insight.E., Labkoff, S., Markel-Fox, S., Tang, P.C., Detmer, D.E. Toward a National Framework for the Secondary Use of Health Data: An American Medical Informatics Association White Paper. Journal of American Medical Informatics Association. 2007;14:1-9.

2. Epilepsy Foundation. Available from: http://Insight.epilepsyfoundation.org/aboutepilepsy/whatisepilepsy/statistics.cfm. (Retrieved on September 24, 2015)

3. Ciechanowski P, Chaytor, N., Miller, J., Fraser, R., Russo, J., Unutzer, J., Gilliam, F. PEARLS Depression Treatment for Individuals with Epilepsy: A Randomized Controlled Trial. Epilepsy \& Behavior. 2010;19(3):225-31.

4. Murphy SN, Weber, G., Mendis, M., Gainer, V., Chueh, Insight. C., Churchill, S., \& Kohane, I. Serving the enterprise and beyond with informatics for integrating biology and the bedside (i2b2). Journal of American Medical Informatics Association. 2010;17(2):124-30.

5. Observational Medical Outcomes Partnership (OMOP) Common Data Model. Available from: http://Insight.ohdsi.org/data-standardization/the-common-datamodel/. (Retrieved on September 24, 2015)

6. Voss EA, Makadia, R., Matcho, A., Ma, Q., Knoll, C., Schuemie M, DeFalco FJ, Londhe A, Zhu V, Ryan PB. Feasibility and utility of applications of the common data model to multiple, disparate observational health databases. Journal of American Medical Informatics Association. 2015.

7. Wilcox A, Vawdrey, D., Weng, C., Velez, M., Bakken, S. Research Data Explorer: Lessons Learned in Design and Development of Context-based Cohort Definition and Selection. American Medical Informaics Association (AMIA) Clinical Research Informatics (CRI) Symposium; San Franscisco2015.

8. Sahoo SS, Lhatoo, S.D., Gupta, D.K., Cui, L., Zhao, M., Jayapandian, C., Bozorgi, A., Zhang, GQ. Epilepsy and seizure ontology: towards an epilepsy informatics infrastructure for clinical research and patient care. Journal of American Medical Informatics Association. 2014;21(1):82-9.

9. DiIorio CK, Bamps, Insight.A., Edwards, A.L., Escoffery, C., Thompson, N.J., Begley, C.E., Shegog, R., Clark, N.M., Selwa, L., Stoll, S.C., Fraser, R.T., Ciechanowski, P., Johnson, E.K., Kobau, R., Price, P.Insight., Managing Epilepsy Well Network. The prevention research centers' managing epilepsy well network. Epilepsy \& Behavior. 2010;19(3):218-24.

10. Sahoo SS, Zhang, GQ., Bamps, Insight., Fraser, R., Stoll, S., Lhatoo, S.D., Tatsuoka, C., Sams, J., Welter, E., Sajatovic, M. Managing information well: Toward an ontology-driven informatics platform for data sharing and secondary use in epilepsy self-management research centers. Health Informatics Journal. 2015:1-14.

11. Rosse C, Mejino Jr., J.L. A reference ontology for biomedical informatics: the Foundational Model of Anatomy. Journal of Biomedical Informatics. 2003;36(2003):478-500.

12. Dou D, Frishkoff, G., Rong, J., Frank, R., Malony, A., Tucker, D., editor. Development of NeuroElectroMagnetic Ontologies (NEMO): A framework for 
mining brain wave ontologies. Thirteenth International Conference on Knowledge Discovery and Data Mining (KDD2007); 2007; San Hose, CA: ACM New York.

13. Nelson SJ, Zeng, K., Kilbourne, J., Powell, T., Moore, R. Normalized names for clinical drugs: RxNorm at 6 years. J Am Med Inform Assoc 2011;18(4):441-8.

14. Cramer JA, Perrine, K., Devinsky, O., Bryant-Comstock, L., Meador, K., Hermann, B. Development and cross-cultural translations of a 31-item quality of life in epilepsy inventory. Epilepsia. 1998;39(1):81-8.

15. Cramer JA, Perrine, K., Devinsky, O., Meador, K. A brief questionnaire to screen for quality of life in epilepsy: the QOLIE-10. Epilepsia. 1996;37(6):577-82.

16. Gershon RC, Lai, J.S., Bode, R., Choi, S., Moy, C., Bleck, T., Miller, D., Peterman, A., Cella, D. Neuro-QOL: quality of life item banks for adults with neurological disorders: item development and calibrations based upon clinical and general population testing. Quality of Life Research. 2012;21(3):475-86.

17. (IOM) CotPHDotE. EPILEPSY across the SPECTRUM. Prmoting Health and Understanding. Washington, DC: 2012.

18. Thurman DJ, Beghi, E., Begley, C.E., Berg, A.T., Buchhalter, J.R., Ding, D., Hesdorffer, D.C., Hauser, Insight.A., Kazis, L., Kobau, R., Kroner, B., Labiner, D., Liow, K., Logroscino, G., Medina, M.T., Newton, C.R., Parko, K., Paschal, A., Preux, P.M., Sander, J.Insight., Selassie, A., Theodore, Insight., Tomson, T., Wiebe, S., ILAE Commission on Epidemiology. Standards for epidemiologic studies and surveillance of epilepsy. Epilepsia. 2011;Suppl 7:2-26.

19. Loring DW, Lowenstein, D.Insight., Barbaro, N.M., Fureman, B.E., Odenkirchen, J., Jacobs, M.P., Austin, J.K., Dlugos, D.J., French, J.A., Gaillard, Insight.D., Hermann, B.P., Hesdorffer, D.C., Roper, S.N., Van Cott, A.C., Grinnon, S., Stout, A. Common data elements in epilepsy research: development and implementation of the NINDS epilepsy CDE project. Epilepsia. 2011;52(6):1186-91.

20. (CDC). Behavioral Risk Factor Surveillance System (BRFSS) 2012. Available from: http://Insight.cdc.gov/brfss/about/brfss today.htm. (Retrieved on September 24, 2015)

21. Horridge M, Bechhofer, S. The OWL API: A Java API for OWL Ontologies. Semantic Web Journal. 2011;2(1):11-21.

22. Centers for Disease Control and Prevention (CDC). Behavioral Risk Factor Surveillance System (BRFSS)—2013 Questionnaire: Section 8, http://Insight.cdc.gov/brfss/about/brfss_today.htm (accessed 20 August 2014).

23. Committee on the Public Health Dimensions of the Epilepsies. EPILEPSY across the SPECTRUM. Promoting health and understanding. Washington, DC: The National Academies Press, 2012.

24. Fisher RS. Emerging antiepileptic drugs. Neurology 1993; 43(suppl. 5): 12-20.

25. Boon P, Vonck K, De Herdt V, et al. Deep brain stimulation in patients with refractory temporal lobe epilepsy. Epilepsia 2007; 48(8): 1551-1560.

26. Begley CE, Famulari M, Annegers JF, et al. The cost of epilepsy in the United States: an estimate from population-based clinical and survey data. Epilepsia 2000; 41(3): 342-351.

27. Chaytor N, Ciechanowski P, Miller JW, et al. Long-term outcomes from the PEARLS randomized trial for the treatment of depression in patients with epilepsy. Epilepsy Behav 2011; 20(3): 545-549. 
28. Aliasgharpour M, Dehgahn Nayeri N, Yadegary MA, et al. Effects of an educational program on selfmanagement in patients with epilepsy. Seizure 2013; 22(1): 48-52.

29. Ciechanowski P, Chaytor N, Miller J, et al. PEARLS depression treatment for individuals with epilepsy: a randomized controlled trial. Epilepsy Behav 2010; 19(3): 225-231.

30. Coleman MT and Newton KS. Supporting self-management in patients with chronic illness. Am Fam Physician 2005; 72(8): 1503-1510.

31. Dilorio C, Escoffery C, Yeager KA, et al. WebEase: development of a Web-based epilepsy selfmanagement intervention. Prev Chronic Dis 2009; 6(1): A28.

32. Dilorio C, Reisinger EL, Yeager KA, et al. A telephone-based self-management program for people with epilepsy. Epilepsy Behav 2009; 14(1): 232-236.

33. Thompson NJ, Walker ER, Obolensky N, et al. Distance delivery of mindfulnessbased cognitive therapy for depression: project UPLIFT. Epilepsy Behav 2010; 19(3): 247-254.

34. Holtzheimer PE, Kelley ME, Gross RE, et al. Subcallosal cingulate deep brain stimulation for treatmentresistant unipolar and bipolar depression. Arch Gen Psychiatry 2012; 69(2): 150-158.

35. Dilorio CK, Bamps YA, Edwards AL, et al.; Managing Epilepsy Well Network. The prevention research centers' managing epilepsy well network. Epilepsy Behav 2010; 19(3): 218-224.

36. Thompson A, Sullivan S, Barley M, et al. Effectiveness of a cognitive behavioural workbook for changing beliefs about antipsychotic polypharmacy: analysis from a cluster randomized controlled trial. J Eval Clin Pract 2010; 16(3): 520-528.

37. Shegog R, Bamps YA, Patel A, et al. Managing Epilepsy Well: emerging e-Tools for epilepsy selfmanagement. Epilepsy Behav 2013; 29(1): 133-140.

38. Nixon A, Kerr C, Breheny K, et al. Patient Reported Outcome (PRO) assessment in epilepsy: a review of epilepsy-specific PROs according to the Food and Drug Administration (FDA) regulatory requirements. Health Qual Life Outcomes 2013; 11 : 38.

39. Patient Reported Outcomes Measurement Information System (PROMIS), http://Insight.nihpromis.org/ (accessed 20 August 2014).

40. Keator DB. Information management in distributed biomedical collaboratories. Methods Mol Biol 2009; 569: 1-23.

41. Helmer KG, Ambite JL, Ames J, et al. Enabling collaborative research using the Biomedical Informatics Research Network (BIRN). J Am Med Inform Assoc 2011; 18(4): 416-422.

42. Gadde S, Aucoin N, Grethe JS, et al. XCEDE: an extensible schema for biomedical data. Neuroinformatics 2012; 10(1): 19-32.

43. Holdren JP and Lander E. Realizing the full potential of health information technology to improve healthcare for Americans: the path forward. Executive Office of the President, Washington, DC, December 2010.

44. Sheth AP and Larson JA. Federated database systems for managing distributed, heterogeneous and autonomous databases. ACM Comput Surv 1990; 22: 183-236.

45. Doan A, Halevy A and Ives Z. Principles of data integration. Waltham, MA: Morgan Kaufmann, 2012.

46. Bodenreider O and Stevens R. Bio-ontologies: current trends and future directions. 
Brief Bioinform 2006; 7(3): 256-274.

47. SNOMED Clinical Terms: The International Health Terminology Standards Development Organisation, http://Insight.nlm.nih.gov/research/umls/Snomed/snomed main.html (accessed 20 August 2014).

48. Cramer JA, Perrine K, Devinsky O, et al. Development and cross-cultural translations of a 31-item quality of life in epilepsy inventory. Epilepsia 1998; 39(1): 81-88.

49. Cramer JA, Perrine K, Devinsky O, et al. A brief questionnaire to screen for quality of life in epilepsy: the QOLIE-10. Epilepsia 1996; 37(6): 577-582.

50. National Institute of Neurological Disorders and Stroke (NINDS). Measuring quality of life in neurological disorders.Final report of the Neuro-QOL study, http://Insight.neuroqol.org/Resources/Resources\%20 documents/NeuroQOLFinal\%20report-2013.pdf (accessed 20 August 2014).

51. Gershon RC, Lai JS, Bode R, et al. Neuro-QOL: quality of life item banks for adults with neurological disorders: item development and calibrations based upon clinical and general population testing. Qual Life Res 2012; 21(3): 475-486.

52. Harris PA, Taylor R, Thielke R, et al. Research electronic data capture (REDCap) - a metadata-driven methodology and workflow process for providing translational research informatics support. J Biomed Inform 2009; 42(2): 377-381.

53. Sahoo SS, Zhang GQ and Lhatoo SD. Epilepsy informatics and an ontology-driven infrastructure for large database research and patient care in epilepsy. Epilepsia 2013; 54(8): 1335-1341. 14 Health Informatics Journal

54. Zhang GQ, Cui L, Lhatoo SD, et al. MEDCIS: multi-modality epilepsy data capture and integration system. In: American Medical Informatics Association (AMIA) annual symposium, Washington, DC, 15-19 November 2014.

55. Sahoo SS, Lhatoo SD, Gupta DK, et al. Epilepsy and seizure ontology: towards an epilepsy informatics infrastructure for clinical research and patient care. J Am Med Inform Assoc 2014; 21(1): 82-89.

56. Thurman DJ, Beghi E, Begley CE, et al. Standards for epidemiologic studies and surveillance of epilepsy. Epilepsia 2011; 52(Suppl. 7): 2-26.

57. Loring DW, Lowenstein DH, Barbaro NM, et al. Common data elements in epilepsy research: development and implementation of the NINDS epilepsy CDE project. Epilepsia 2011; 52(6): 1186-1191.

58. Loddenkemper T, Kellinghaus C, Wyllie E, et al. A proposal for a five-dimensional patient oriented epilepsy classification. Epileptic Disord 2005; 7: 308-316.

59. Kellinghaus C, Loddenkemper T, Wyllie E, et al. Suggestion for a new, patientoriented epilepsy classification. Nervenarzt 2006; 77: 961-969.

60. Bodenreider O, Peters L and Nguyen T. RxNav: Browser and application programming interfaces for drug information sources. In: AMIA annual symposium, Washington, DC, 22-26 October 2011, p. 2129.

61. Sahoo SS, Zhao M, Luo L, et al. OPIC: ontology-driven patient information capturing system for epilepsy. In: The American Medical Informatics Association (AMIA) annual symposium, Chicago, IL, 3-7 November 2012.

62. Cui L, Bozorgi A, Lhatoo SD, et al. EpiDEA: extracting structured epilepsy and seizure information from patient discharge summaries for cohort identification. In: The American Medical Informatics Association (AMIA) annual symposium, 
Chicago, IL, 3-7 November 2012.

63. Sahoo SS, Jayapandian C, Garg G, et al. Heartbeats in the cloud: distributed analysis of electrophysiological "Big Data" using cloud computing for epilepsy clinical research. J Am Med Inform Assoc 2014; 21(2): 263-271.

64. Halevy A, Rajaraman A and Ordille JJ (eds). Data integration: the teenage years. In: Proceedings of the 32nd international conference on Very Large Data Bases (VLDB), Seoul, Korea, 12-15 September 2006. VLDB Endowment.

65. Lebo T, Sahoo SS and McGuinness D. PROV-O: the PROV ontology. World Wide Web consortium W3C Recommendation, 30 April 2013.

66. Fielding RT and Taylor RN. Principled design of the modern Web architecture. ACM T Internet Techn 2002; 2(2): 115-150.

67. Zhang GQ, Siegler T, Saxman P, et al. VISAGE: a query interface for clinical research. In: AMIA clinical research informatics summit, San Francisco, CA, 12-13 March 2010, pp. 76-80.

68. HBase: The Hadoop database, http://hbase.apache.org/ (Retrieved on November 1, 2015)

69. Ruby on Rails: http://api.rubyonrails.org (Retrieved on November 1, 2015)

70. Ruby on Rails: http://guides.rubyonrails.org/getting_started.html (Retrieved on November 1, 2015)

71. MySQL: http://Insight.oracle.com/us/products/mysql/mysqlstandard/overview/index.html (Retrieved on November 1, 2015)

72. HighCharts: http://Insight.highcharts.com/products/highcharts (Retrieved on November 1, 2015)

73. Ruby Version Manager: https://rvm.io (Retrieved on November 1, 2015)

74. https://Insight.phusionpassenger.com (Retrieved on November 1, 2015)

75. CSS: http://Insight.w3.org/Style/CSS/Overview.en.html (Retrieved on November 1, 2015)

76. http://getbootstrap.com (Retrieved on November 1, 2015) 